\title{
Tuning nonequilibrium heat current and two-photon statistics via composite qubit-resonator interaction
}

\author{
Ze-Huan Chen, ${ }^{1}$ Han-Xin Che, ${ }^{1}$ Zhe-Kai Chen, ${ }^{1}$ Chen Wang,,${ }^{1, *}$ and Jie Ren ${ }^{2, \dagger}$ \\ ${ }^{1}$ Department of Physics, Zhejiang Normal University, Jinhua 321004, Zhejiang, People's Republic of China \\ ${ }^{2}$ Center for Phononics and Thermal Energy Science, China-EU Joint Center for Nanophononics, Shanghai Key Laboratory of Special \\ Artificial Microstructure Materials and Technology, School of Physics Sciences and Engineering, Tongji University, Shanghai 200092, China
}

(Received 9 October 2021; accepted 20 January 2022; published 23 February 2022)

\begin{abstract}
Quantum thermal transport and two-photon statistics serve as two representative nonequilibrium features in circuit quantum electrodynamics (cQED) systems. Here we investigate quantum heat flow and two-photon correlation function at steady state in a composite qubit-resonator model, where one qubit shows both transverse and longitudinal couplings to a single-mode optical resonator. With weak qubit-resonator interaction, we unravel two microscopic transport pictures, i.e., cotunneling and cyclic heat exchange processes corresponding to transverse and longitudinal couplings, respectively. The nonmonotonic behavior of the heat current is exhibited by tuning the temperature bias with the weak longitudinal coupling. At strong qubit-resonator coupling, the heat current also exhibits a nonmonotonic feature by increasing qubit-resonator coupling strength, which tightly relies on the scattering processes between the qubit and the corresponding thermal bath. Furthermore, the longitudinal coupling is preferred to enhance heat current in the strong qubit-resonator coupling regime. For two-photon correlation function, it exhibits an antibunching-to-bunching transition by tuning the composite angle, which is mainly dominated by the modulation of the energy gap between the first and the second excited eigenstates. Our results are expected to deepen the understanding of nonequilibrium thermal transport and nonclassical photon radiation based on the cQED platform.
\end{abstract}

DOI: 10.1103/PhysRevResearch.4.013152

\section{INTRODUCTION}

Deep understanding and efficient characterization of nonequilibrium excitation processes via quantum light-matter interactions constitute an active frontier for quantum optics and quantum transport [1-5]. Heat flow is considered as one generic feature of quantum thermal transport, which is bounded by the second law of thermodynamics. Under thermodynamic bias (e.g., voltage and temperature bias), the heat current is driven directionally from the hot source to the cold drain. However, the direction of the current can be reversed against the thermodynamic bias, e.g., by quantum correlations [6] and geometric-phase-induced pump [7-9].

Due to the dramatic advancement of quantum circuit technology, the circuit quantum electrodynamics (cQED) systems emerge as one promising platform to realize quantum light-matter interactions [10-13]. The cQED systems are traditionally described by the seminal quantum Rabi model (QRM) [14-17], i.e., one two-level qubit transversely interacting with a single-mode photon resonator, which is

\footnotetext{
*Corresponding author: wangchenyifang@gmail.com; wangchen@zjnu.cn

${ }^{\dagger}$ Corresponding author: Xonics@tongji.edu.cn

Published by the American Physical Society under the terms of the Creative Commons Attribution 4.0 International license. Further distribution of this work must maintain attribution to the author(s) and the published article's title, journal citation, and DOI.
}

able to describe ultrastrong qubit-resonator coupling [18-20]. QRM has been extensively investigated in finite-component quantum phase transition [21-24], quantum nonlinear optics [25-28], and quantum thermodynamics [29-31].

As one main characteristic of nonequilibrium excitation processes in CQED systems, quantum thermal transport has attracted increasing attention recently, which leads to a flurry of valuable works [4,32-36]. Particularly, Ronzani et al. [4] experimentally detected heat flow in a hybrid quantum system comprising one transmon-qubit and two microwave resonators, of which the resonators are individually coupled to two metallic resistors, respectively. Consequently, the typical thermal functionalities were realized, e.g., heat valve [4], thermal diode [32], and thermal transistor [33,36]. Moreover, Iles-Smith et al. [37,38] and Maguire et al. [39] theoretically investigated Franco-Condon physics in noncommutative QRM via the reaction coordinate mapping approach. Yamamoto and Kato [40] unraveled a nontrivial two-peak feature of thermal conductance in QRM at the linear response limit. Wang et al. [41-43] analyzed nonmonotonic behavior of the heat current in a longitudinally coupled qubitresonator model. However, for the influence of the composite qubit-photon interaction on nonequilibrium heat flow and the microscopic picture in dissipative qubit-resonator hybrid systems, e.g., QRM, there currently is a lack of exploration, which is crucial to deepen the understanding of nonequilibrium heat transport based on the $\mathrm{CQED}$ platform.

Meanwhile, nonclassical photon radiation is considered as another representative probe of nonequilibrium 
excitation processes in hybrid quantum systems [44-46], which is mainly characterized by two-photon correlation function. It is known that two-photon correlation function was initially proposed by R. J. Glauber to unveil the optical coherence of quantum theory [47], which has later been extensively applied to investigate nonequilibrium correlations of photons, e.g., superradiant spontaneous emission [48-53], the strong interaction of photons [44,54], and the dissipative phase transition [55]. Rabl [56], Ridolfo et al. [57,58], Stassi et al. [59], and Garziano et al. [60] proposed a modified definition of two-photon correlation function under the dressed basis to properly characterize photon nonclassicality in the optomechanics and open QRM, respectively, which is valid even at strong qubit-photon coupling. Consequently, Schaeverbeke et al. [61] applied such dressed expression of two-photon correlation function to connect single photon emission with the Franck-Condon blockade effect. Bin et al. [62,63] introduced the concept of $N$-phonon bundle emission. For the cQED systems, an alternative scheme, i.e., longitudinal coupling between the qubit and the resonator, can also be realized based on the superconducting circuit engineering $[64,65]$, which has pronounced consequences for nonclassical-photon-state generation [66-68], scalable circuit design [69,70], and fast nondemolition qubit readout [71,72]. However, though both the transverse and the longitudinal coupling limits can be flexibly modulated [26,64-67,73], the influence of composite qubit-resonator interaction on two-photon statistics has not yet been reported.

In this paper, we applied dressed master equation (DME) to investigate the effect of composite qubit-resonator interaction on quantum thermal transport. At weak qubit-resonator coupling, it is found that the heat current exhibits monotonic enhancement by increasing thermal bath temperature bias in the dissipative QRM, which stems from cotunneling transport processes. In contrast, the current is changed to show a nonmonotonic feature with longitudinal qubit-resonator coupling, which is dominated by cyclic heat exchange transitions. These two distinct microscopic processes are crucial to unraveling the physics pictures of quantum thermal transport in dissipative cQED systems. At strong qubit-resonator coupling with different composite angles, the current generally shows nomonotonic behavior, which tightly relies on the scattering processes between the qubit and the bosons in the corresponding thermal bath. Furthermore, the optimal composite angle to generate the maximal heat current gradually changes from the transverse coupling type to the longitudinal counterpart with an increase of qubit-resonator coupling strength. We also study steady-state two-photon correlation function at strong qubit-resonator coupling. It is found that by tuning up the composite angle, an antibunching-to-bunching transition is significantly exhibited, which mainly originates from increment and reduction of the energy gap between the first and the second excited states. In particular, a giant bunching signature of photons is unraveled at moderate composite angle.

The rest of this paper is organized as follows: In Sec. II, we present the composite qubit-resonator model, derive the DME, and obtain the expression of the steady-state heat current. In Sec. III, we investigate the effect of the composite angle on the steady-state heat current and two-photon correlation
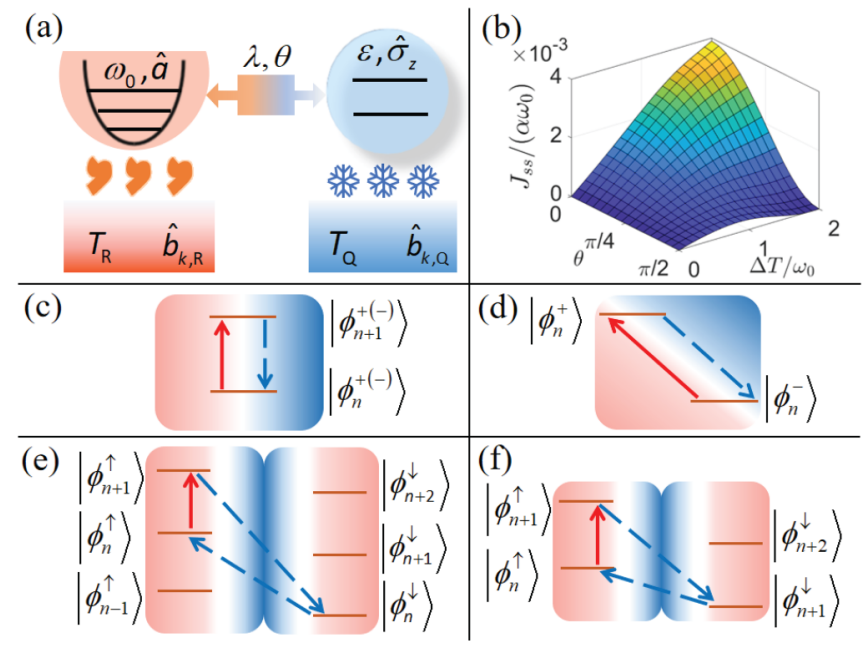

FIG. 1. (a) The schematic description of a composite qubitresonator model. The red half circle (top left) is the optical resonator, where $\hat{a}$ annihilates one photon with the frequency $\omega_{0}$. The blue circle (top right) represents the qubit, which is characterized by the Pauli operator $\hat{\sigma}_{z}$ and the splitting energy $\varepsilon$. The double-arrowed rectangle shows the composite qubit-resonator interaction with the interaction strength $\lambda$ and composite angle $\theta$. The red (bottomleft) and blue (bottom-right) rectangles denote two thermal baths, characterized by temperatures $T_{\mathrm{R}}$ and $T_{\mathrm{Q}}$ and bosonic annihilators $\hat{b}_{k, \mathrm{R}}$ and $\hat{b}_{k, \mathrm{Q}}$. (b) Steady-state heat current $J_{s s} /\left(\alpha \omega_{0}\right)$ modulated by temperature bias $\Delta T / \omega_{0}$ and the composite angle $\theta$, with weak qubit-resonator interaction strength $\lambda=0.01 \omega_{0}$. Other parameters are given by $\varepsilon=1.5 \omega_{0}, \alpha=0.001, \omega_{c}=10 \omega_{0}, T_{\mathrm{R}}=\omega_{0}+\Delta T / 2$, and $T_{\mathrm{Q}}=\omega_{0}-\Delta T / 2$. (c) and (d) show cotunneling processes for first terms of the components $I_{x, 1}$ and $I_{x, 2}$ in Eqs. (17a) and (17b), respectively. Panels (e) and (f) describe cyclic energy exchange processes for first terms of current components $I_{z, 1}$ and $I_{z, 2}$ in Eqs. (19a) and (19b), respectively. The red solid (blue dashed) arrowed lines denote transitions between two eigenstates assisted by the R-th (Qth) thermal bath.

function. The microscopic pictures of these behaviors are also discussed. Finally, we give a summary in Sec. IV.

\section{MODEL AND METHOD}

\section{A. Composite qubit-resonator model}

We study the dissipative qubit-resonator hybrid model in Fig. 1(a), where one two-level qubit shows both longitudinal and transverse couplings to a single-mode resonator, each individually interacting with the corresponding thermal baths. The Hamiltonian is described as $\left(\hbar=1\right.$ and $\left.k_{B}=1\right)$

$$
\hat{H}=\hat{H}_{\mathrm{S}}^{\theta}+\sum_{\mu=\mathrm{Q}, \mathrm{R}}\left(\hat{H}_{\mathrm{B}}^{\mu}+\hat{V}_{\mu}\right) .
$$

Specifically, the composite qubit-resonator system is expressed as

$$
\hat{H}_{\mathrm{S}}^{\theta}=\frac{\varepsilon}{2} \hat{\sigma}_{z}+\omega_{0} \hat{a}^{\dagger} \hat{a}+\lambda\left(\cos \theta \hat{\sigma}_{x}+\sin \theta \hat{\sigma}_{z}\right)\left(\hat{a}^{\dagger}+\hat{a}\right),
$$

where $\hat{a}^{\dagger}(\hat{a})$ is the creation (annihilation) operator of one photon in the resonator with the frequency $\omega_{0}, \varepsilon$ is the splitting energy of the two-level qubit; $\hat{\sigma}_{\alpha}(\alpha=x, y, z)$ is the 
Pauli operators of the qubit under the qubit basis $\{\uparrow, \downarrow\}$, with $\hat{\sigma}_{z}|\uparrow\rangle=|\uparrow\rangle$ and $\hat{\sigma}_{z}|\downarrow\rangle=-|\downarrow\rangle ; \lambda$ is the qubit-resonator coupling strength; and $\theta$ is the angle to tune the composite qubit-resonator interaction. $\hat{H}_{\mathrm{B}}^{\mu}=\sum_{k} \omega_{k, \mu} \hat{b}_{k, \mu}^{\dagger} \hat{b}_{k, \mu}$ describes the $\mu$-th bosonic thermal bath, where $\hat{b}_{k, \mu}^{\dagger}\left(\hat{b}_{k, \mu}\right)$ creates (annihilates) one boson with the frequency $\omega_{k, \mu}$ and momentum $k$. The system-bath interactions are described as

$$
\begin{aligned}
& \hat{V}_{\mathrm{R}}=\left(\hat{a}^{\dagger}+\hat{a}\right) \sum_{k} g_{k, \mathrm{R}}\left(\hat{b}_{k, \mathrm{R}}^{\dagger}+\hat{b}_{k, \mathrm{R}}\right), \\
& \hat{V}_{\mathrm{Q}}=\hat{\sigma}_{x} \sum_{k} g_{k, \mathrm{Q}}\left(\hat{b}_{k, \mathrm{Q}}^{\dagger}+\hat{b}_{k, \mathrm{Q}}\right),
\end{aligned}
$$

with $g_{k, \mu}$ the coupling strength. The $\mu$-th thermal bath is characterized by the spectral function $\gamma_{\mu}(\omega)=$ $2 \pi \sum_{k}\left|g_{k, \mu}\right|^{2} \delta\left(\omega-\omega_{k, \mu}\right)$. In this work, we specify the spectral function as the Ohmic case, i.e., $\gamma_{\mu}(\omega)=$ $\pi \alpha \omega \exp \left(-|\omega| / \omega_{c}\right)$, with $\alpha$ the dissipation strength and $\omega_{c}$ the cutoff frequency.

Generally, it is difficult to analytically find the eigensolution of the qubit-resonator hybrid system at Eq. (2). However, in the limiting case we may obtain the analytical expression. Specifically, at $\theta=0$ the composite system with only transverse coupling is simplified as

$$
\hat{H}_{\mathrm{S}}^{0}=\frac{\varepsilon}{2} \hat{\sigma}_{z}+\omega_{0} \hat{a}^{\dagger} \hat{a}+\lambda \hat{\sigma}_{x}\left(\hat{a}^{\dagger}+\hat{a}\right)
$$

which is the seminal QRM $[14,15]$. Hence the eigenvalues can be mapped to the roots of transcendental $G$ function by applying the Bargmann algebra and extended coherent states approaches [16,17], respectively. In weak qubit-resonator coupling regime, the QRM is reduced to the Jaynes-Cummings model $\hat{H}_{\mathrm{JC}}=\varepsilon \hat{\sigma}_{z} / 2+\omega_{0} \hat{a}^{\dagger} \hat{a}+\lambda\left(\hat{a}^{\dagger} \hat{\sigma}_{-}+\right.$ $\hat{a} \hat{\sigma}_{+}$), which is dominated by the rotating-wave terms. Consequently, the eigenvalues are given by

$$
E_{n, \pm}=(n+1 / 2) \omega_{0} \pm \sqrt{\left(\varepsilon-\omega_{0}\right)^{2} / 4+\lambda^{2}(n+1)},
$$

and the corresponding eigenvectors are

$$
\begin{aligned}
& \left|\phi_{n}^{+}\right\rangle=\cos \frac{\theta_{n}}{2}|n, \uparrow\rangle+\sin \frac{\theta_{n}}{2}|n+1, \downarrow\rangle, \\
& \left|\phi_{n}^{-}\right\rangle=-\sin \frac{\theta_{n}}{2}|n, \uparrow\rangle+\cos \frac{\theta_{n}}{2}|n+1, \downarrow\rangle,
\end{aligned}
$$

with $\tan \theta_{n}=2 \lambda \sqrt{n+1} /\left(\varepsilon-\omega_{0}\right)$.

While at $\theta=\pi / 2$ the qubit is longitudinally coupled to the resonator $[41,66,67,69,70,73]$, with the Hamiltonian

$$
\hat{H}_{\mathrm{S}}^{\pi / 2}=\frac{\varepsilon}{2} \hat{\sigma}_{z}+\omega_{0} \hat{a}^{\dagger} \hat{a}+\lambda \hat{\sigma}_{z}\left(\hat{a}^{\dagger}+\hat{a}\right) .
$$

Accordingly, the eigenvalues are expressed as

$$
\begin{aligned}
& E_{n, \uparrow}=\omega_{0} n+\varepsilon / 2-\lambda^{2} / \omega_{0}, \\
& E_{n, \downarrow}=\omega_{0} n-\varepsilon / 2-\lambda^{2} / \omega_{0},
\end{aligned}
$$

and the eigenvectors are described by the extended coherent boson states

$$
\begin{aligned}
& \left|\phi_{n}^{\uparrow}\right\rangle=\exp \left[\frac{\lambda}{\omega_{0}}\left(\hat{a}-\hat{a}^{\dagger}\right)\right] \frac{\left(\hat{a}^{\dagger}\right)^{n}}{\sqrt{n !}}|0\rangle_{a} \otimes|\uparrow\rangle, \\
& \left|\phi_{n}^{\downarrow}\right\rangle=\exp \left[-\frac{\lambda}{\omega_{0}}\left(\hat{a}-\hat{a}^{\dagger}\right)\right] \frac{\left(\hat{a}^{\dagger}\right)^{n}}{\sqrt{n !}}|0\rangle_{a} \otimes|\downarrow\rangle,
\end{aligned}
$$

with the vacuum state of the resonator $\hat{a}|0\rangle_{a}=0$.
Recently, tunable photonic heat transport and thermal functionalities (e.g., thermal diode and quantum thermal transistor) have been experimentally realized in superconducting cQED devices [4,32-34]. The superconducting qubit (e.g., transmon qubit) is able to show either the longitudinal or the transverse interaction with the photon resonator $[18,19,64$ 67]. Moreover, the R-th bath, i.e., $\hat{H}_{\mathrm{B}}^{\mathrm{R}}=\sum_{k} \omega_{k, \mathrm{R}} \hat{b}_{k, \mathrm{R}}^{\dagger} \hat{b}_{k, \mathrm{R}}$, may correspond to one metallic resistor, e.g., a thin-film copper microstrip resistor with several superconducting aluminium probes $[4,32]$ or a general noiseless resistor with a fluctuating voltage source [33,74]. The noisy resistor may produce the continuous-modes bosonic noise (e.g., electromagnetic noise), quantified by the temperature $T_{\mathrm{R}}$. While the Q-th thermal bath interacting with the qubit, i.e., $\hat{H}_{\mathrm{B}}^{\mathrm{Q}}=$ $\sum_{k} \omega_{k, \mathrm{Q}} \hat{b}_{k, \mathrm{Q}}^{\dagger} \hat{b}_{k, \mathrm{Q}}$, could be alternatively simulated by an LC circuit coupled to one Ohmic resistor, quantified by the temperature $T_{\mathrm{Q}}[33,75]$. The electromagnetic noise should pass the LC circuit to affect the qubit. Such effective construction of the Q-th thermal bath makes the energy exchange between the qubit and the corresponding bath available. Hence the dissipative composite qubit-resonator model Eq. (1) in this work could be realized based on the circuit QED platforms.

We also compare the present model (1) with the qubitbiased open QRM [76-78]

$$
\begin{aligned}
\hat{H}_{\text {dissip-Rabi }}= & \varepsilon^{\prime} \hat{\sigma}_{z} / 2+\Delta \hat{\sigma}_{x} / 2+\omega_{0} \hat{a}^{\dagger} \hat{a}+\lambda \hat{\sigma}_{z}\left(\hat{a}^{\dagger}+\hat{a}\right) \\
& +\sum_{k, \mu=\mathrm{R}, \mathrm{Q}} \omega_{k, \mu} \hat{b}_{k, \mu}^{\dagger} \hat{b}_{k, \mu} \\
& +\left(\hat{a}^{\dagger}+\hat{a}\right) \sum_{k} g_{k, \mathrm{R}}\left(\hat{b}_{k, \mathrm{R}}^{\dagger}+\hat{b}_{k, \mathrm{R}}\right) \\
& +\hat{\sigma}_{z} \sum_{k} g_{k, \mathrm{Q}}\left(\hat{b}_{k, \mathrm{Q}}^{\dagger}+\hat{b}_{k, \mathrm{Q}}\right),
\end{aligned}
$$

with $\Delta$ being the qubit bias strength. For the closed composite qubit-resonator model (2), we can reexpress it as

$$
\hat{H}_{\mathrm{S}}^{\theta}=\frac{\varepsilon}{2} \sin \theta \hat{\tau}_{z}-\frac{\varepsilon}{2} \cos \theta \hat{\tau}_{x}+\omega_{0} \hat{a}^{\dagger} \hat{a}+\lambda \hat{\tau}_{z}\left(\hat{a}^{\dagger}+\hat{a}\right),
$$

where $\hat{\tau}_{z}=|+\rangle\langle+|-|-\rangle\langle-|$ and $\hat{\tau}_{x}=|+\rangle\langle-|+|-$ \rangle$+\mid$, under the basis $|+\rangle=\left[\left(\cos \frac{\theta}{2}+\sin \frac{\theta}{2}\right)|\uparrow\rangle+\left(\cos \frac{\theta}{2}-\right.\right.$ $\left.\left.\sin \frac{\theta}{2}\right)|\downarrow\rangle\right] / \sqrt{2}$ and $|-\rangle=\left[-\left(\cos \frac{\theta}{2}-\sin \frac{\theta}{2}\right)|\uparrow\rangle+\left(\cos \frac{\theta}{2}+\right.\right.$ $\left.\left.\sin \frac{\theta}{2}\right)|\downarrow\rangle\right] / \sqrt{2}$. Hence $\hat{H}_{\mathrm{S}}^{\theta}$ is equivalent with the longitudinally coupled QRM [79-81] by mapping $\varepsilon \sin \theta \rightarrow \varepsilon^{\prime}$ and $-\varepsilon \cos \theta \rightarrow \Delta$. However, by including system-bath quantum dissipation, it is found that under such transformation of Eq. (11), the qubit-bath interaction (3b) will be modified to

$$
\hat{V}_{\mathrm{Q}}=\left(\cos \theta \hat{\tau}_{z}+\sin \theta \hat{\tau}_{x}\right) \sum_{k} g_{k, \mathrm{Q}}\left(\hat{b}_{k, \mathrm{Q}}^{\dagger}+\hat{b}_{k, \mathrm{Q}}\right),
$$

which is generally distinct from the qubit-bath coupling in the open QRM in Eq. (10), stemming from the appearance of the additional term $\hat{V}_{\text {add }}=\sin \theta \hat{\tau}_{x} \sum_{k} g_{k, \mathrm{Q}}\left(\hat{b}_{k, \mathrm{Q}}^{\dagger}+\right.$ $\hat{b}_{k, \mathrm{Q}}$ ). This additional term may have non-negligible influence on the dissipative dynamics and transport behaviors of the qubit-resonator hybrid system. Particularly in the strong coupling limit, the qubit-photon interaction dominates $\hat{H}_{\mathrm{S}}^{\theta}$, which leads to the approximate eigenstates, i.e., $\left|\phi_{n}^{ \pm}\right\rangle \approx \exp [ \pm \lambda(\hat{a}-$ $\left.\left.\hat{a}^{\dagger}\right) / \omega_{0}\right] \frac{\left(\hat{a}^{\dagger}\right)^{n}}{\sqrt{n !}}|0\rangle_{a} \otimes| \pm\rangle$. Thus $\hat{V}_{\text {add }}$ may dominate the energy 
exchange processes between the qubit and the Q-th thermal bath, which is characterized as the spin flip accompanied with the coherent eigenstates transitions.

\section{B. Quantum master equation}

We consider weak interactions between the composite hybrid system and the bosonic thermal baths. We focus on the steady-state properties of the composite qubit-resonator system, where the off-diagonal elements of the reduced system density operator in the eigenbasis of $\hat{H}_{\mathrm{S}}$ become negligible. Thus $\hat{V}_{\mathrm{R}}$ and $\hat{V}_{\mathrm{Q}}$ at Eqs. (3a) and (3b) can be properly perturbed. Under the Born approximation, the total density operator can be separated as $\hat{\rho}_{\text {tot }}(t) \approx \hat{\rho}_{\mathrm{S}}(t) \otimes \hat{\rho}_{\mathrm{B}, \mathrm{R}} \otimes \hat{\rho}_{\mathrm{B}, \mathrm{Q}}$, where $\hat{\rho}_{\mathrm{S}}(t)$ is the reduced hybrid system density operator, and $\hat{\rho}_{\mathrm{B}, \mu}=\exp \left(-\hat{H}_{\mathrm{B}}^{\mu} / k_{\mathrm{B}} T_{u}\right) / \operatorname{Tr}_{\mathrm{B}}\left\{\exp \left(-\hat{H}_{\mathrm{B}}^{\mu} / k_{\mathrm{B}} T_{u}\right)\right\}(\mu=\mathrm{R}, \mathrm{Q})$ is the density operator of the $\mu$-th thermal bath, with $k_{\mathrm{B}}$ the Boltzmann constant and $T_{\mu}$ is the temperature of the $\mu$-th bath. Then, by further including the Markovian approximation, we obtain the quantum DME as $[20,82,83]$

$$
\begin{aligned}
\frac{d}{d t} \hat{\rho}_{\mathrm{S}}(t)= & i\left[\hat{\rho}_{\mathrm{S}}(t), \hat{H}_{\mathrm{S}}^{\theta}\right]+\sum_{n, m, \mu}\left\{\Gamma_{\mu}^{+}\left(E_{n m}\right) \hat{\mathcal{L}}_{n m}\left[\hat{\rho}_{\mathrm{S}}(t)\right]\right. \\
& \left.+\Gamma_{\mu}^{-}\left(E_{n m}\right) \hat{\mathcal{L}}_{m n}\left[\hat{\rho}_{\mathrm{S}}(t)\right]\right\},
\end{aligned}
$$

where the dissipator is given by $\hat{\mathcal{L}}_{n m}\left[\hat{\rho}_{\mathrm{S}}(t)\right]=$ $\left|\psi_{n}\right\rangle\left\langle\psi_{m}\left|\hat{\rho}_{\mathrm{S}}(t)\right| \psi_{m}\right\rangle\left\langle\psi_{n}\right|-\left(\left|\psi_{m}\right\rangle\left\langle\psi_{m}\right| \hat{\rho}_{\mathrm{S}}(t)+\right.$ $\left.\hat{\rho}_{\mathrm{S}}(t)\left|\psi_{m}\right\rangle\left\langle\psi_{m}\right|\right) / 2$, with $\hat{H}_{\mathrm{S}}^{\theta}\left|\psi_{n}\right\rangle=E_{n}\left|\psi_{n}\right\rangle$, and the corresponding transitions rates are given by

$$
\begin{aligned}
& \Gamma_{\mu}^{+}\left(E_{n, m}\right)=\gamma_{\mu}\left(E_{n, m}\right) n_{\mu}\left(E_{n, m}\right)\left|\left\langle\psi_{n}\left|\hat{A}_{\mu}\right| \psi_{m}\right\rangle\right|^{2}, \\
& \Gamma_{\mu}^{-}\left(E_{n, m}\right)=\gamma_{\mu}\left(E_{n m}\right)\left[1+n_{\mu}\left(E_{n, m}\right)\right]\left|\left\langle\psi_{n}\left|\hat{A}_{\mu}\right| \psi_{m}\right\rangle\right|^{2},
\end{aligned}
$$

with $n_{\mu}\left(E_{n, m}\right)=1 /\left[\exp \left(E_{n, m} / T_{u}\right)-1\right]$ the Bose-Einstein distribution function, $\hat{A}_{\mathrm{R}}=\hat{a}^{\dagger}+\hat{a}, \hat{A}_{\mathrm{Q}}=\hat{\sigma}_{x}, E_{n, m}=E_{n}-E_{m}$ the energy gap between two eigenstates $\left|\psi_{n}\right\rangle$ and $\left|\psi_{m}\right\rangle$ of $\hat{H}_{\mathrm{S}}$. The rate $\Gamma_{\mu}^{+(-)}\left(E_{n, m}\right)$ describes the energy exchange process that the composite qubit-resonator system is excited (relaxed) from the eigenstate $\left|\psi_{m}\right\rangle$ to $\left|\psi_{n}\right\rangle$ by absorbing (releasing) one photon with the energy $E_{n}-E_{m}$ from (into) the $\mu$-th thermal bath.

Therefore, after long-time evolution, i.e., $d \hat{\rho}_{\mathrm{S}}(t) / d t=0$, we can obtain the steady-state population distribution $P_{n}$ with $P_{n}=\left\langle\psi_{n}\left|\hat{\rho}_{\mathrm{S}}(t \rightarrow \infty)\right| \psi_{n}\right\rangle$. Moreover, from the DME Eq. (13) we obtain the steady-state heat current into the Q-th bath (see Sec. A of the Appendix)

$$
J_{s s}=\sum_{E_{n}>E_{n^{\prime}}} E_{n, n^{\prime}}\left[\Gamma_{\mathrm{Q}}^{-}\left(E_{n, n^{\prime}}\right) P_{n}-\Gamma_{\mathrm{Q}}^{+}\left(E_{n, n^{\prime}}\right) P_{n^{\prime}}\right],
$$

where the energy gap is $E_{n, n^{\prime}}=E_{n}-E_{n^{\prime}}$.

\section{RESULTS AND DISCUSSIONS}

\section{A. Steady-state heat current}

\section{Weak qubit-resonator interaction}

Heat flow is considered the representative transport feature of nonequilibrium excitation processes in dissipative qubit-resonator hybrid systems. Here we first investigate the steady-state behavior of heat current at weak qubit-resonator coupling in Fig. 1(b), which is modulated by both the temperature bias $\Delta T=T_{\mathrm{R}}-T_{\mathrm{Q}}$ and the composite angle $\theta$. It is found that for small $\theta$, the heat current exhibits a monotonic increase by increasing the temperature bias $\Delta T$, particularly in the limit of $\theta=0$, i.e., the dissipative QRM. However, in the large $\theta$ regime, the heat current is changed to shown nonmonotonic behavior, i.e., the current is first enhanced and later suppressed with the increase of $\Delta T$, which identifies the signature of the negative differential thermal conductance (NDTC) [84-88]. The appearance of NDTC is consistent with previous works $[41,42]$. It needs to be noted that although it is not shown here, a similar result can also be found at resonance $\left(\varepsilon=\omega_{0}\right)$. Hence we conclude that the composite qubit-resonator interaction strongly affects the steady-state heat current.

Then we try to explore microscopic processes of heat transport with weak qubit-resonator coupling. We admit that to analytically find the microscopic mechanism with arbitrary composite angle $\theta$ is quite difficult. Here we focus on two limits, i.e., $\theta=0$ and $\theta=\pi / 2$, to unravel the representative physical pictures of the heat current. For $\theta=0$, under the eigenbasis $\left\{\left|\phi_{n}^{ \pm}\right\rangle\right\}$of QRM in Eqs. (6a) and (6b), the leading order of steady-state heat current at finite energy bias regime [e.g., $\left(\varepsilon-\omega_{0}\right) \gg \lambda$ ] can be analytically expressed as (see the detail in Sec. B of the Appendix)

$$
J_{s s}^{x} \approx \frac{\lambda^{2}}{\left(\varepsilon-\omega_{0}\right)^{2}}\left(\omega_{0} I_{x, 1}+\varepsilon I_{x, 2}\right),
$$

where two components are specified as

$$
I_{x, 1}=\gamma_{\mathrm{Q}}\left(\omega_{0}\right)\left\{n_{\mathrm{R}}\left(\omega_{0}\right)\left[1+n_{\mathrm{Q}}\left(\omega_{0}\right)\right]-\left[1+n_{\mathrm{R}}\left(\omega_{0}\right)\right] n_{\mathrm{Q}}\left(\omega_{0}\right)\right\},
$$

$I_{x, 2}=\frac{\gamma_{\mathrm{R}}(\varepsilon)}{2 n_{\mathrm{Q}}(\varepsilon)+1}\left\{n_{\mathrm{R}}(\varepsilon)\left[1+n_{\mathrm{Q}}(\varepsilon)\right]-\left[1+n_{\mathrm{R}}(\varepsilon)\right] n_{\mathrm{Q}}(\varepsilon)\right\}$.

Both $I_{x, 1}$ and $I_{x, 2}$ are dominated by cotunneling processes. Specifically, $I_{x, 1}$ describes the process such that as the state $\left|\phi_{n}^{\eta}\right\rangle\left(\left|\phi_{n+1}^{\eta}\right\rangle\right)$ is excited (relaxed) to $\left|\phi_{n+1}^{\eta}\right\rangle\left(\left|\phi_{n}^{\eta}\right\rangle\right)$ by absorbing (emitting) energy $\omega_{0}$ from (into) the R-th reservoir, the transition $\left|\phi_{n+1}^{\eta}\right\rangle \rightarrow\left|\phi_{n}^{\eta}\right\rangle\left(\left|\phi_{n}^{\eta}\right\rangle \rightarrow\left|\phi_{n+1}^{\eta}\right\rangle\right)$ simultaneously occurs by emitting (absorbing) $\omega_{0}$ into (from) the Q-th reservoir with $\eta= \pm$, which is also shown in Fig. 1(c). While $I_{x, 2}$ shows other typical cotunneling processes, exemplified in Fig. 1(d), the excitation (relaxation) transition $\left|\phi_{n}^{-(+)}\right\rangle \rightarrow\left|\phi_{n}^{+(-)}\right\rangle$by absorbing (emitting) energy $\varepsilon$ from (into) the R-th reservoir is accompanied by the dual transition $\left|\phi_{n}^{+(-)}\right\rangle \rightarrow\left|\phi_{n}^{-(+)}\right\rangle$. It is interesting to find that the directional cotunneling transport from the R-th reservoir to that of the Q-th one, i.e., described by first terms of $I_{x, 1}$ and $I_{x, 2}$, is monotonically enhanced with increase of the temperature bias, which mainly contribute to $J_{s s}^{x}$ at finite temperature bias. In contrast, the opposite transitions (from the Q-th reservoir to that of the R-th one) are dramatically suppressed. Finally, the current in Eq. (16) becomes significant at large temperature bias $\left(T_{\mathrm{R}} \approx 2 \omega_{0}, T_{\mathrm{Q}} \approx 0\right)$, which is specified as $J_{s s}^{x} \approx\left[\lambda /\left(\varepsilon-\omega_{0}\right)\right]^{2}\left[\omega_{0} \gamma_{\mathrm{Q}}\left(\omega_{0}\right) n_{\mathrm{R}}\left(\omega_{0}\right)+\varepsilon \gamma_{\mathrm{R}}(\varepsilon) n_{\mathrm{R}}(\varepsilon)\right]$.

For $\theta=\pi / 2$, it is found that the transition coefficient $\left\langle\phi_{n}^{\sigma}\left|\hat{\sigma}_{x}\right| \phi_{n^{\prime}}^{\bar{\sigma}}\right\rangle$ in Eqs. (14a) and (14b) under the coherentstate basis $\left\{\left|\phi_{n}^{\uparrow(\downarrow)}\right\rangle\right\}$ is approximated as $\left\langle\phi_{n}^{\uparrow}\left|\hat{\sigma}_{x}\right| \phi_{n^{\prime}}^{\downarrow}\right\rangle \approx$ 
$(-1)^{n}\left[\delta_{n, n^{\prime}}+\left(2 \lambda / \omega_{0}\right)\left(\sqrt{n+1} \delta_{n, n^{\prime}-1}-\sqrt{n} \delta_{n, n^{\prime}+1}\right)\right]$ (also see Refs. $[89,90]$ ). Based on the systematic perturbation (see the full solution in Sec. C of the Appendix), the steady-state heat current in the bias regime $\left[\left(\varepsilon-\omega_{0}\right) \gg \lambda\right]$ is described as

$$
J_{s s}^{z} \approx\left(\frac{2 \lambda}{\omega_{0}}\right)^{2} \omega_{0}\left(I_{z, 1}+I_{z, 2}\right),
$$

where these two components are given by

$$
\begin{aligned}
I_{z, 1}= & \frac{\gamma_{\mathrm{Q}}\left(\varepsilon+\omega_{0}\right)}{2 n_{\mathrm{Q}}(\varepsilon)+1}\left\{\left[1+n_{\mathrm{Q}}\left(\varepsilon+\omega_{0}\right)\right] n_{\mathrm{Q}}(\varepsilon) n_{\mathrm{R}}\left(\omega_{0}\right)\right. \\
& \left.-n_{\mathrm{Q}}\left(\varepsilon+\omega_{0}\right)\left[1+n_{\mathrm{Q}}(\varepsilon)\right]\left[1+n_{\mathrm{R}}\left(\omega_{0}\right)\right]\right\}, \\
I_{z, 2}= & \frac{\gamma_{\mathrm{Q}}\left(\varepsilon-\omega_{0}\right)}{2 n_{\mathrm{Q}}(\varepsilon)+1}\left\{\left[1+n_{\mathrm{Q}}(\varepsilon)\right] n_{\mathrm{Q}}\left(\varepsilon-\omega_{0}\right) n_{\mathrm{R}}\left(\omega_{0}\right)\right. \\
& \left.-n_{\mathrm{Q}}(\varepsilon)\left[1+n_{\mathrm{Q}}\left(\varepsilon-\omega_{0}\right)\right]\left[1+n_{\mathrm{R}}\left(\omega_{0}\right)\right]\right\} .
\end{aligned}
$$

In sharp contrast to $\theta=0$ limit, $I_{z, 1}$ is contributed by two competing cyclic fluxes. Specifically, the first term $\left[1+n_{\mathrm{Q}}\left(\varepsilon+\omega_{0}\right)\right] n_{\mathrm{Q}}(\varepsilon) n_{\mathrm{R}}\left(\omega_{0}\right)$ describes the loop transition $\left|\phi_{n+1}^{\uparrow}\right\rangle \rightarrow\left|\phi_{n}^{\downarrow}\right\rangle \rightarrow\left|\phi_{n}^{\uparrow}\right\rangle \rightarrow\left|\phi_{n+1}^{\uparrow}\right\rangle$ by directionally transferring the energy $\omega_{0}$ into the Q-th thermal reservoir, which is also depicted in Fig. 1(c). The second term $n_{\mathrm{Q}}(\varepsilon+$ $\left.\omega_{0}\right)\left[1+n_{\mathrm{Q}}(\varepsilon)\right]\left[1+n_{\mathrm{R}}\left(\omega_{0}\right)\right]$ shows the counter loop transition. Similarly, $I_{z, 2}$ is composed of other, two opposite cyclic fluxes, where the first loop path is shown in Fig. 1(d) and characterizes the joint transport process $\left|\phi_{n+1}^{\uparrow}\right\rangle \rightarrow\left|\phi_{n+1}^{\downarrow}\right\rangle \rightarrow\left|\phi_{n}^{\uparrow}\right\rangle \rightarrow\left|\phi_{n+1}^{\uparrow}\right\rangle$. Intriguingly, at large temperature bias $\Delta T \approx 2 \omega_{0}$, i.e., $T_{\mathrm{R}} \approx 2 \omega_{0}$ and $T_{\mathrm{Q}} \approx 0$, all cyclic current components in $I_{z, 1}$ and $I_{z, 2}$ break down due to negligible excitation in the Q-th reservoir $\left[n_{\mathrm{Q}}(\omega>0) \approx 0\right]$. This directly results in the suppression of the steady-state heat current, which identifies the emergence of the NDTC.

Therefore, we exploit two distinct microscopic pictures in limiting composite angles with weak qubit-resonator interaction, i.e., cotunneling transitions at $\theta=0$ and cyclic transitions at $\theta=\pi / 2$, which are generic to unraveling microscopic mechanisms of quantum thermal transport, e.g., in the nonequilibrium spin-boson model [91-96], metal-insulator interfaces [97,98], and inelastic thermoelectrics [99-103]. The analytical expressions of the heat current in Eqs. (16) and (18) are obtained for the first time in a dissipative qubit-resonator hybrid model. Recently, the NDTC was also reported in a longitudinal and quadratic qubit-resonator model [43] based on the analysis of transition rates. The transition between squeezed eigenstates, assisted by the spin-flip processes, allows only two or zero squeezed photons excitation (relaxation). In contrast, the corresponding transition in the longitudinal and linear qubit-resonator model (i.e., $\theta=\pi / 2$ ) allows at most one coherent photon change. Though the transition trajectories are distinct for such two hybrid models, these two microscopic transitions establish analogous thermodynamic cycles of heat transfer, which both lead to the emergence of the NDTC.

\section{Strong qubit-resonator interaction}

Next we investigate steady-state heat current $J_{s s} /\left(\alpha \omega_{0}\right)$ beyond weak qubit-resonator coupling with typical composite angles in Fig. 2. As the qubit shows a transverse

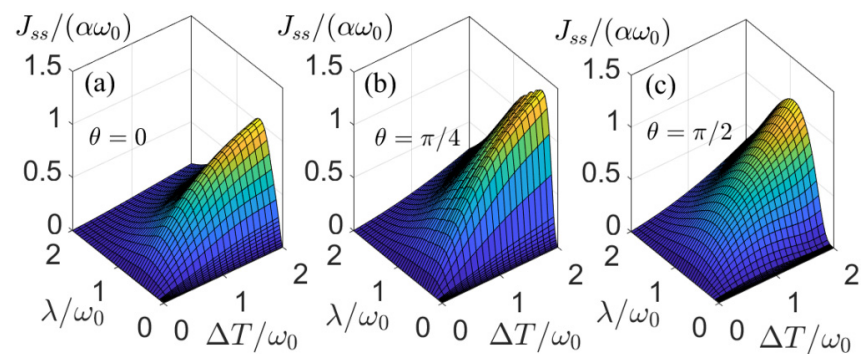

FIG. 2. Steady-state heat current $J_{s s} /\left(\alpha \omega_{0}\right)$ modulated by temperature bias $\Delta T / \omega_{0}$ and qubit-resonator interaction strength $\lambda / \omega_{0}$, with composite angle (a) $\theta=0$, (b) $\theta=\pi / 4$, and (c) $\theta=\pi / 2$. Other parameters are given by $\varepsilon=1.5 \omega_{0}, \alpha=0.001, \omega_{c}=10 \omega_{0}$, $T_{\mathrm{R}}=\omega_{0}+\Delta T / 2$, and $T_{\mathrm{Q}}=\omega_{0}-\Delta T / 2$.

interaction with the optical resonator, i.e., $\theta=0$ in Fig. 2(a), the heat current exhibits nonmonotonic behavior by increasing qubit-resonator interaction strength under finite temperature bias (e.g., $\Delta T / \omega_{0}=1$ ). Due to the effect of counter-rotatingterms, the eigenstates of the QRM beyond Eqs. (6a) and (6b) will introduce additional energy exchange transitions, which may effectively enhance the heat current in the regime $\lambda / \omega_{0} \lesssim 0.5$. While in strong qubit-resonator coupling limit (e.g., $\left.\lambda / \omega_{0}>1.5\right)$, the eigenstates become nearly degenerate, i.e., $\left|\phi_{n}^{ \pm}\right\rangle \approx \exp \left[ \pm \lambda\left(\hat{a}-\hat{a}^{\dagger}\right) / \omega_{0}\right]\left[\left(\hat{a}^{\dagger}\right)^{n} / \sqrt{n !}\right]|0\rangle \otimes| \pm\rangle$ with $\hat{\sigma}_{x}| \pm\rangle= \pm| \pm\rangle$, which significantly prohibits energy exchange between the hybrid system and the Q-th reservoir $\left(\left\langle\phi_{n}^{+}\left|\hat{\sigma}_{x}\right| \phi_{n}^{-}\right\rangle \approx 0\right)$. Then, by tuning on the composite angle, e.g., $\theta=\pi / 4$ and $\pi / 2$, it is found that the profiles of heat currents modulated by $\Delta T / \omega_{0}$ and $\lambda / \omega_{0}$ in Figs. 2(b) and 2(c) are generally similar with limiting angle case $\theta=0$. In particular for the limiting case $\theta=\pi / 2$, the transition coefficient in Eqs. (14a) and (14b) is expressed as $[89,90]$

$$
\begin{aligned}
\left\langle\phi_{n}^{\uparrow}\left|\hat{\sigma}_{x}\right| \phi_{n^{\prime}}^{\downarrow}\right\rangle= & (-1)^{n} \exp \left(-2 \lambda^{2} / \omega_{0}^{2}\right) \sqrt{n ! n^{\prime} !} \\
& \times \sum_{l=0}^{\min \left[n, n^{\prime}\right]} \frac{(-1)^{l} \sqrt{\left(2 \lambda / \omega_{0}\right)^{n+n^{\prime}-2 l}}}{(n-l) !\left(n^{\prime}-l\right) ! l !},
\end{aligned}
$$

which induces higher-order transitions between $\left|\phi_{n}^{\uparrow(\downarrow)}\right\rangle$ and $\left|\phi_{n^{\prime}}^{\downarrow(\uparrow)}\right\rangle$ with $\left|n-n^{\prime}\right| \geqslant 2$ besides the lowest-order transport processes, i.e., cyclic exchange in Figs. 1(e) and 1(f). These additional transitions are robust even at large temperature bias, which mainly results in comparatively large heat current and the disappearance of the NDTC. While at finite temperature bias (e.g., $\left.\Delta T / \omega_{0}=1\right)$, the initial enhancement of $J_{s s} /\left(\alpha \omega_{0}\right)$ by increasing $\lambda / \omega_{0}$ stems from additional transport processes. It may be quantified by $\left\langle\phi_{n}^{\uparrow}\left|\hat{\sigma}_{x}\right| \phi_{n^{\prime}}^{\downarrow}\right\rangle \approx(-1)^{n}\{[1-$ $\left.(n+1 / 2)\left(\frac{2 \lambda}{\omega_{0}}\right)^{2}\right] \delta_{n, n^{\prime}}+\left(\frac{2 \lambda}{\omega_{0}}\right)\left(\sqrt{n+1} \delta_{n, n^{\prime}-1}-\sqrt{n} \delta_{n, n^{\prime}+1}\right)+$ $\left.\frac{1}{2}\left(\frac{2 \lambda}{\omega_{0}}\right)^{2}\left[\sqrt{n(n-1)} \delta_{n, n^{\prime}+2}+\sqrt{(n+1)(n+2)} \delta_{n, n^{\prime}-2}\right]\right\}$, where the last two terms will enhance the current by forming efficient transition paths. However, the final decrease of heat current in the strong qubit-resonator coupling limit is mainly attributed to the fact that the transition coefficient $\left\langle\phi_{n}^{\uparrow}\left|\hat{\sigma}_{x}\right| \phi_{n^{\prime}}^{\downarrow}\right\rangle$ in Eq. (20) and the corresponding transition rates are dramatically weakened. Consequently, the energy exchange processes are strongly blocked. 


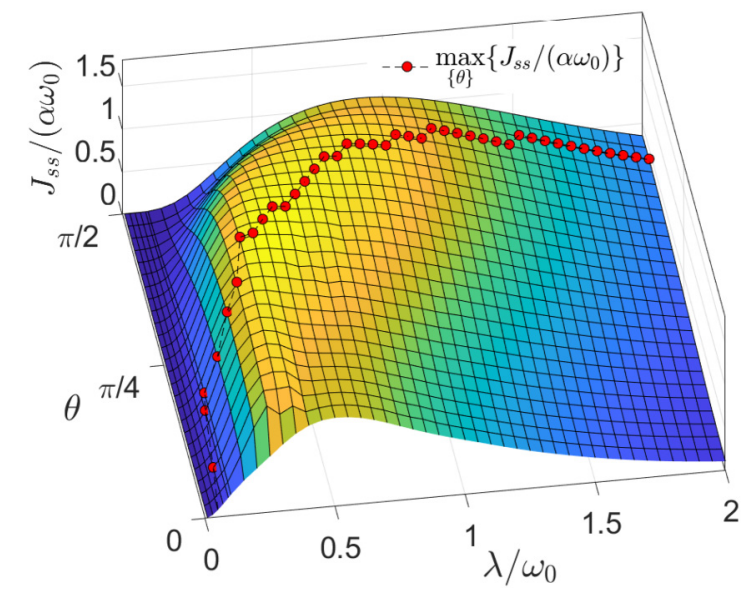

FIG. 3. Steady-state heat current $J_{s s} /\left(\alpha \omega_{0}\right)$ modulated by qubitresonator interaction strength $\lambda / \omega_{0}$ and composite angle $\theta$. The dashed line with red circles shows the maximal $J_{s s} /\left(\alpha \omega_{0}\right)$ by searching $\theta \in[0, \pi / 2]$ with given $\lambda / \omega_{0}$. Other parameters are given by $\varepsilon=1.5 \omega_{0}, \alpha=0.001, \omega_{c}=10 \omega_{0}, T_{\mathrm{R}}=2 \omega_{0}$, and $T_{\mathrm{Q}}=0$.

Moreover, we analyze the interplay between the composite angle and the qubit-resonator coupling strength on $\max _{\{\theta\}}\left\{J_{s S} /\left(\alpha \omega_{0}\right)\right\}$ in Fig. 3. It is intriguing to find that with an increase of the qubit-resonator interaction, the composite angle dominating the maximal heat current is gradually modified from 0 to $\pi / 2$, corresponding to the transverse and longitudinal qubit-resonator couplings, respectively. In particular for the strong qubit-photon coupling limit, the eigenspace of the Hamiltonian of the quantum hybrid system (2) is dominated by coherent eigenstates with large displacement, which is approximated as $\hat{H}_{\mathrm{S}}^{\theta} \approx \omega_{0} \hat{a}^{\dagger} \hat{a}+\lambda\left(\cos \theta \hat{\sigma}_{x}+\sin \theta \hat{\sigma}_{z}\right)\left(\hat{a}^{\dagger}+\hat{a}\right)$. If we set $\theta=0$, it is known that $\left[\hat{H}_{\mathrm{S}}^{\theta}, \hat{V}_{\mathrm{Q}}\right] \approx 0$. This demonstrates that the energy exchange between the qubit and the Q-th bath becomes negligible, which leads to the vanish of the heat flow. While as $\theta=\pi / 2$, the transitions between coherent eigenstates [(9a) and (9b)] assisted by the spin-flipinvolved energy exchange are finite, which results in the heat current larger than the counterpart with the transverse coupling. Hence we conclude that at strong $\lambda / \omega_{0}$, the longitudinal coupling type is preferred to enhance the steady-state heat current.

\section{B. Two-photon correlation function}

Nonclassical photon radiation, as the main correlation probe of nonequilibrium excitation processes of photons in the dissipative qubit-photon hybrid systems, is typically quantified by two-photon correlation function. Two-photon correlation function describes the correlation between two temporally separated photon signals from one light source, which is pioneered by R. J. Glauber to exhibit the optical coherence of quantum theory [47]. Alternatively, Rabi [56], Ridolfo et al. [57,58], Stassi et al. [59], and Garziano et al. [60] proposed a modified definition of two-photon correlation function within the dressed picture. Hence it can also be safely included to study photon statistics in the present model in Eq. (1) with strong qubit-resonator interaction. Specifically, two-photon correlation function at steady state is defined

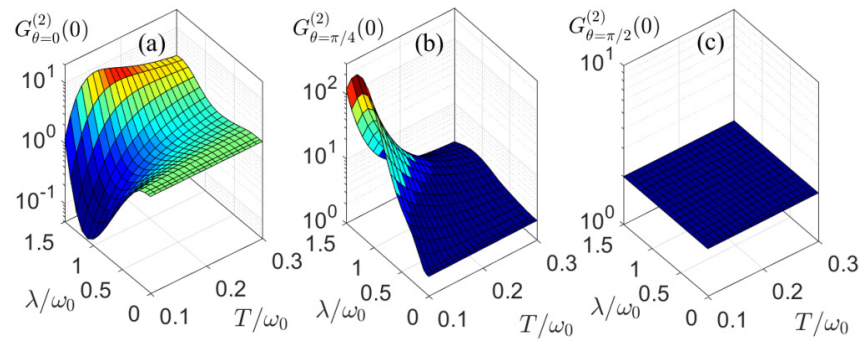

FIG. 4. Two-photon correlation function as a function of qubitresonator interaction strength $\lambda / \omega_{0}$ and temperature $T_{\mathrm{R}}=T_{\mathrm{Q}}=T$, with typical composite angle (a) $\theta=0$, (b) $\theta=\pi / 4$, and (c) $\theta=$ $\pi / 2$. Other parameters are given by $\varepsilon=1.5 \omega_{0}, \alpha=0.001$, and $\omega_{c}=$ $10 \omega_{0}$.

as

$$
G_{\theta}^{(2)}(\tau)=\lim _{t \rightarrow \infty} \frac{\left\langle\hat{X}_{\theta}^{+}(t) \hat{X}_{\theta}^{+}(t+\tau) \hat{X}_{\theta}^{-}(t+\tau) \hat{X}_{\theta}^{-}(t)\right\rangle}{\left\langle\hat{X}_{\theta}^{+}(t) \hat{X}_{\theta}^{-}(t)\right\rangle^{2}}
$$

where the measurement operator is $\hat{X}_{\theta}^{-}=-i \sum_{k>j} \Delta_{k j} X_{j k}$ $\left|\psi_{j}\right\rangle\left\langle\psi_{k}\right|$ and $\hat{X}_{\theta}^{+}=\left(\hat{X}_{\theta}^{-}\right)^{\dagger}$, with $X_{j k}=\left\langle\psi_{j}\left|\left(\hat{a}^{\dagger}+\hat{a}\right)\right| \psi_{k}\right\rangle$ the energy-gap $\Delta_{j k}=E_{j}-E_{k}$ and the eigensolution $\hat{H}_{\mathrm{S}}^{\theta}\left|\psi_{k}\right\rangle=$ $E_{k}\left|\psi_{k}\right\rangle$. Here we focus on the effect of the composite qubit-resonant interaction on zero-time two-photon correlation function $G_{\theta}^{(2)}(0)$.

We first investigate two-photon correlation function by tuning both the qubit-resonator coupling strength and the bath temperature with different composite angle in Fig. 4. For $\theta=0$, it is found that an intriguing antibunching behavior can be found with strong $\lambda$, which demonstrates the seminal two-photon blockade [57]. Then, by tuning on the composite angle, e.g., $\theta=\pi / 4$, a giant photon bunching behavior is exhibited at low temperature. If we further increase the composite angle to $\theta=\pi / 2$, it is shown that $G_{\theta=0}^{(2)}(0) \approx 2$ regardless of $\lambda$ and $T\left(T_{\mathrm{R}}=T_{\mathrm{Q}}=T\right)$ due to the fully thermalization of the longitudinally coupled qubit-resonator system

$$
\begin{aligned}
\rho_{\mathrm{S}}(\infty)= & \frac{\sinh \left[\omega_{0} /(2 T)\right]}{\cosh [\varepsilon /(2 T)]} \sum_{n} e^{-\frac{(n+1 / 2) \omega_{0}}{T}} \\
& \left.\times\left[e^{-\frac{\varepsilon}{2 T}}\left|\phi_{n}^{\uparrow}\right\rangle\left\langle\phi_{n}^{\uparrow}\right|+e^{\frac{\varepsilon}{2 T}}\right]\left|\phi_{n}^{\downarrow}\right\rangle\left\langle\phi_{n}^{\downarrow}\right|\right] .
\end{aligned}
$$

Moreover, we plot Fig. 5(a) to see the influence of the composite angle on two-photon correlation function. It is found that at strong $\lambda$ (e.g., $\lambda / \omega_{0} \approx 1$ ), an antibunching-to-bunching transition is clearly exhibited by increasing $\theta$. Therefore we conclude that the modulation of the composite angle is quite important to exhibit the nonclassical photon statistics, which may provide physical guidance to measure photon correlation in circuit QED.

Next we analyze the mechanism of antibunching-tobunching transition modulated by the composite angle. At low temperature (e.g., $T=0.1 \omega_{0}$ ), the finite spacing distribution of energy levels [see Fig. 5(b)] results in $P_{0} \gg P_{1} \gg P_{2} \gg P_{3}$, exhibited in Fig. 5(c). Hence one-photon and two-photon terms are approximated as $\left\langle\hat{X}_{\theta}^{+} \hat{X}_{\theta}^{-}\right\rangle \approx P_{1} A_{1}$ and $\left\langle\left(\hat{X}_{\theta}^{+}\right)^{2}\left(\hat{X}_{\theta}^{-}\right)^{2}\right\rangle \approx P_{2} B_{2}$, where the coefficients are $A_{n}=$ $\sum_{l<k}\left(\Delta_{k l} X_{k l}\right)^{2}$ and $B_{n}=\sum_{p<l<k}\left(\Delta_{k l} \Delta_{l p} X_{k l} X_{l p}\right)^{2}$. Consequently, the two-photon correlation function is expressed in 


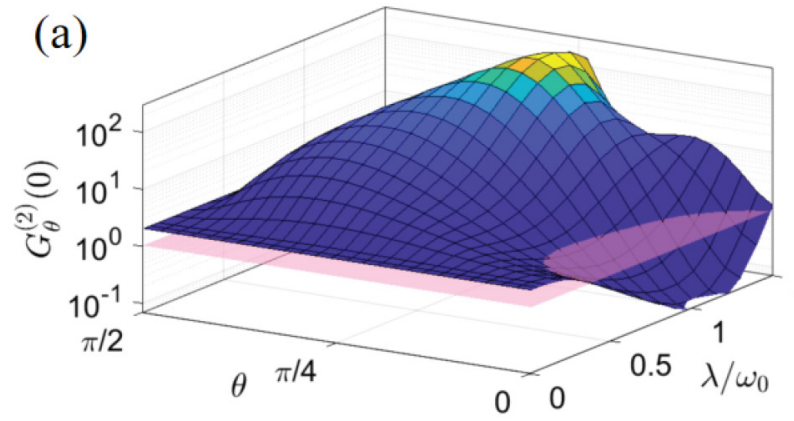

(b)
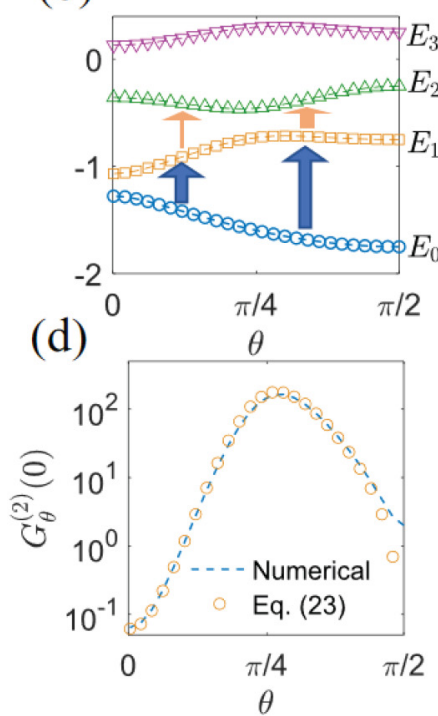

(c)

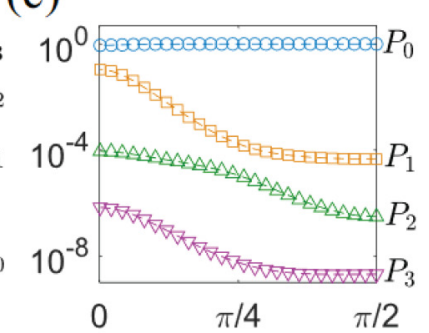

(e)

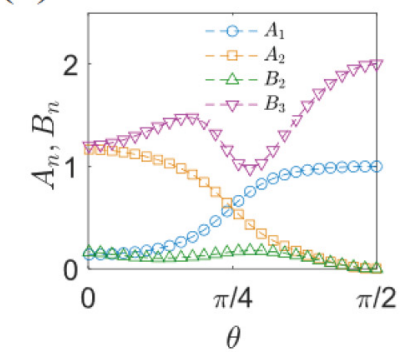

FIG. 5. (a) Two-photon correlation function $G_{\theta}^{(2)}(0)$ modulated by qubit-resonator interaction strength $\lambda / \omega_{0}$ and composite angle $\theta$ in low-temperature regime, (b) comparison of $G_{\theta}^{(2)}(0)$ via numerical calculation with that based on Eq. (23), (c) four lowest eigenenergies, (d) four lowest steady-state populations, and (e) coefficients $A_{n}, B_{n}$ as a function of composite angle $\theta$ at $\lambda=\omega_{0}$. Other parameters are given by $\varepsilon=1.5 \omega_{0}, \alpha=0.001, \omega_{c}=10 \omega_{0}$, and $T_{\mathrm{R}}=T_{\mathrm{Q}}=0.1 \omega_{0}$.

a concise way,

$$
G_{\theta}^{(2)}(0) \approx \frac{P_{2} B_{2}}{\left(P_{1} A_{1}\right)^{2}},
$$

which shows agreement with the numerical result in Fig. 5(d) in a wide regime of $\theta$ at strong qubit-resonator coupling $\left(\lambda / \omega_{0}=1\right)$. Particularly in the antibunching regime (e.g., $0<\theta \lesssim \pi / 10)$, the large energy-gap $\left(E_{2}-E_{1}\right)$ suppresses the ratio $P_{2} / P_{1}^{2}$. Moreover, the coefficients $A_{1}$ and $B_{2}$ are nearly flat, as shown in Fig. 5(e). Hence the transition $\left|\psi_{1}\right\rangle \rightarrow\left|\psi_{2}\right\rangle$ is strongly blocked, leading to the antibunching behavior of photons. While in the bunching regime (e.g., $\pi / 10 \lesssim \theta \leqslant \pi / 4$ ), though $A_{1}$ is strengthened by increasing $\theta$, the reduction of energy gap between $E_{2}$ and $E_{1}$ dramatically enhances steadystate population $P_{2}$ and successive two-photon excitation process $\left|\psi_{0}\right\rangle \rightarrow\left|\psi_{1}\right\rangle \rightarrow\left|\psi_{2}\right\rangle$, resulting in the bunching behavior of photons, as shown in Fig. 5(b). Moreover, we analyze the behavior of steady-state two-photon correlation function by individually modulating $T_{\mathrm{R}}$ and $T_{\mathrm{Q}}$ in Fig. 6. When $\theta=0$, i.e., the transverse qubit-photon coupling, it is shown that increasing either $T_{\mathrm{R}}$ or $T_{\mathrm{Q}}$ suppresses photon antibunching. By
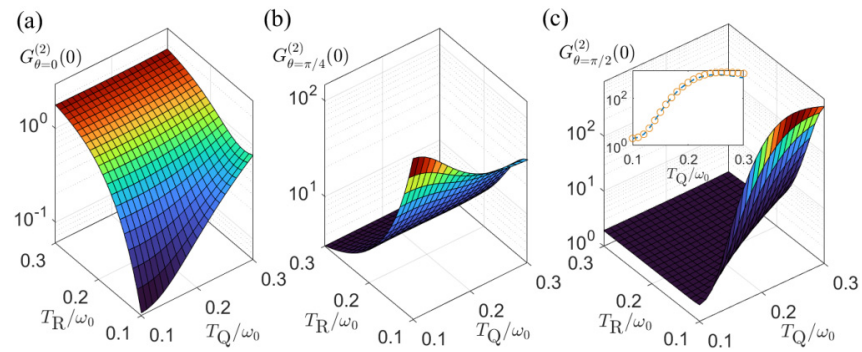

FIG. 6. Two-photon correlation function $G_{\theta}^{(2)}(0)$ as a function of two bath temperatures $T_{\mathrm{R}}$ and $T_{\mathrm{Q}}$, with (a) $\theta=0$, (b) $\theta=\pi / 4$, and (c) $\theta=\pi / 2$. The inset in (c) shows the comparison of Eq. (24) with the numerical result. Other parameters are given by $\varepsilon=1.5 \omega_{0}, \lambda=$ $\omega_{0}, \alpha=0.001$, and $\omega_{c}=10 \omega_{0}$.

tuning on the composite angle, e.g., $\theta=\pi / 4$, the increase of $T_{\mathrm{R}}$ dramatically reduces the photon bunching signature. While in the longitudinal qubit-photon coupling limit, i.e., $\theta=\pi / 2$, it is intriguing to observe a giant photon bunching behavior as $T_{\mathrm{R}} \approx 0.1 \omega_{0}$ and $T_{\mathrm{Q}}$ exceeds $T_{\mathrm{R}}$. Under the truncated basis $\left\{\left|\psi_{0}\right\rangle=\left|\phi_{0}^{\downarrow}\right\rangle,\left|\psi_{1}\right\rangle=\left|\phi_{1}^{\downarrow}\right\rangle,\left|\psi_{2}\right\rangle=\left|\phi_{0}^{\uparrow}\right\rangle,\left|\psi_{3}\right\rangle=\left|\phi_{2}^{\downarrow}\right\rangle\right\}$, the photon correlation measurement-induced transition is blocked $\left|\psi_{1}\right\rangle \nrightarrow\left|\psi_{2}\right\rangle\left(X_{12}=0\right)$. Consequently, the correlation term $\left\langle\left(\hat{X}_{\pi / 2}^{+}\right)^{2}\left(\hat{X}_{\pi / 2}^{-}\right)^{2}\right\rangle$ is contributed by two-photon excitation process $\left|\psi_{0}\right\rangle \rightarrow\left|\psi_{1}\right\rangle \rightarrow\left|\psi_{3}\right\rangle$. Then, the two-photon correlation function can be estimated as

$$
G_{\pi / 2}^{(2)}(0) \approx \frac{2 P_{3}}{P_{1}^{2}},
$$

which shows agreement with the numerical result in the inset of Fig. 6(c). By tuning $T_{\mathrm{Q}}$ above $T_{\mathrm{R}}$, it is found that two successive spin-flip-involved scattering processes, i.e., $\left|\psi_{0}\right\rangle \rightarrow\left|\psi_{1}\right\rangle$ and $\left|\psi_{1}\right\rangle \rightarrow\left|\psi_{3}\right\rangle$, significantly excite the steadystate population $P_{3}$ compared with $P_{1}$, which leads to the emergence of the giant photon bunching feature.

\section{CONCLUSION}

In summary, we investigate the effect of composite qubitresonator interaction on quantum thermal transport and two-photon correlation function at steady state. We apply the quantum DME to properly treat strong qubit-resonator interaction with arbitrary composite angle. For heat transport at weak qubit-resonator coupling, it is found that the heat current with transverse qubit-resonator coupling shows monotonic behavior by increasing bath temperature bias. It is dominated by the cotunneling process, which is quantified by Eqs. (17a) and (17b), while the current is gradually changed to exhibit nonmonotonic feature by tuning on the composite angle, signifying the emergence of the NDTC effect. Such a NDTC effect is characterized as cyclic energy exchange processes and described in Eqs. (19a) and (19b). Hence we unravel two crucial microscopic processes for quantum thermal transport. While in the strong qubit-resonator interaction regime, the heat current with $\theta=0$ exhibits nonmonotonic behavior by increasing qubit-resonator interaction strength. The initial enhancement of $J_{S S}$ stems from additional energy transitions due to counter-rotating terms, whereas the final suppression of $J_{s s}$ is attributed to the nearly degeneracy of eigenstates, which 
prohibits energy exchange between the qubit and the Q-th thermal reservoir. Moreover, it is intriguing to find that the optimal composite angle, which corresponds to the maximal heat current, switches directionally from $\theta=0$ (transverse) to $\theta=\pi / 2$ (longitudinal) by modulating the qubit-resonator interaction from weak to strong couplings. Hence longitudinal coupling is preferred to enhance the steady-state heat current in the strong qubit-resonator interaction regime.

We also investigate the steady-state two-photon correlation function by modulating the composite angle. At $\theta=0$, the pronounced anti-bunching feature is exhibited in the regime of low temperature and strong qubit-resonator interaction. Then by tuning on $\theta$, we find the giant bunching signal instead. By further increasing $\theta$ (e.g., $\theta=\pi / 2$ ), two-photon correlation function is globally around 2 due to thermal distribution of the density operator of the qubit-resonator hybrid system in Eq. (22). Furthermore, we present the mechanism with approximate expression in Eq. (23) to explain this antibunching-to-bunching transition. The antibunching and bunching behaviors of photons are modulated by the enlarged and reduced energy gap between the first and the second excited eigenstates, respectively. Moreover, we analyze steady-state two-photon correlation function by individually tuning two bath temperatures. With $\theta=\pi / 2$, the giant photon bunching behavior is observed as $T_{\mathrm{Q}}>T_{\mathrm{R}}$. The spin-flipinvolved scattering processes dramatically enhance photon bunching correlation. We hope that our results affected by composite qubit-resonator interaction may deepen the understanding of quantum thermal transport and two-photon statistics in dissipative QED systems.

\section{ACKNOWLEDGMENTS}

Z.-H.C., H.-X.C., Z.-K. C., and C.W. are supported by the National Natural Science Foundation of China under Grant No. 11704093 and the Opening Project of Shanghai Key Laboratory of Special Artificial Microstructure Materials and Technology. J.R. acknowledges the support by the National Natural Science Foundation of China (No. 11935010 and No. 11775159), Natural Science Foundation of Shanghai (No. 18ZR1442800 and No. 18JC1410900).

\section{APPENDIX}

\section{General expression of heat current}

From the quantum DME Eq. (13), the population dynamics is described as

$$
\frac{d}{d t} \rho_{n}(t)=\sum_{n, m, \mu}\left[\Gamma_{\mu}^{-}\left(E_{n, m}\right) \rho_{m}(t)-\Gamma_{\mu}^{+}\left(E_{n, m}\right) \rho_{n}(t)\right],
$$

with the transition rates $\Gamma_{\mu}^{ \pm}\left(E_{n, m}\right)$ specified by Eqs. (14a) and (14b), and the population $\rho_{n}(t)=\left\langle\phi_{n}\left|\hat{\rho}_{\mathrm{S}}(t)\right| \phi_{n}\right\rangle$. Hence the expression of steady-state heat current into the $\mu$-th thermal bath is expressed as

$$
J_{\mu}=\sum_{E_{n}>E_{n^{\prime}}} E_{n, n^{\prime}}\left[\Gamma_{\mu}^{-}\left(E_{n, n^{\prime}}\right) P_{n}-\Gamma_{\mu}^{+}\left(E_{n, n^{\prime}}\right) P_{n^{\prime}}\right] .
$$

Then, we try to approximately investigate the steady state populations $P_{n}=\left\langle\phi_{n}\left|\hat{\rho}_{\mathrm{S}}(t \rightarrow \infty)\right| \phi_{n}\right\rangle$ at weak qubit-resonator coupling limit via the perturbation method. As we reexpress the populations in the vector form $\left|\rho_{s}\right\rangle$, the steady-state solution based on Eq. (A1) becomes $\mathcal{L}\left|\rho_{s}\right\rangle=0$. At weak qubitresonator coupling, we expand $\left|\rho_{\mathrm{S}}\right\rangle \approx\left|\rho_{\mathrm{S}}^{(0)}\right\rangle+\left(\lambda / \omega_{0}\right)^{2}\left|\rho_{\mathrm{S}}^{(1)}\right\rangle$ and $\mathcal{L} \approx \mathcal{L}^{(0)}+\left(\lambda / \omega_{0}\right)^{2} \mathcal{L}^{(1)}$ up to $\left(\lambda / \omega_{0}\right)^{2}$. Hence the general solution is given by

$$
\begin{aligned}
\mathcal{L}^{(0)}\left|\rho_{\mathrm{S}}^{(0)}\right\rangle & =0, \\
\mathcal{L}^{(0)}\left|\rho_{\mathrm{S}}^{(1)}\right\rangle+\mathcal{L}^{(1)}\left|\rho_{\mathrm{S}}^{(0)}\right\rangle & =0 .
\end{aligned}
$$

\section{2. $\theta=0$ case}

At $\theta=0$, the model in the weak qubit-resonator coupling regime becomes the dissipative Jaynes-Cummings model. We consider the off-resonant regime $\left[\left(\varepsilon-\omega_{0}\right) \gg \lambda\right]$. Under the eigenbasis $\left|\phi_{n}^{ \pm}\right\rangle$[Eqs. (6a) and (6b)] the transition coefficients involved with the Q-th bath are specified as

$$
\begin{aligned}
\left\langle\phi_{n+1}^{+}\left|\hat{\sigma}_{x}\right| \phi_{n}^{+}\right\rangle & =\sin \frac{\varphi_{n}}{2} \cos \frac{\varphi_{n+1}}{2}, \\
\left\langle\phi_{n+1}^{+}\left|\hat{\sigma}_{x}\right| \phi_{n}^{-}\right\rangle & =\cos \frac{\varphi_{n}}{2} \cos \frac{\varphi_{n+1}}{2}, \\
\left\langle\phi_{n+1}^{-}\left|\hat{\sigma}_{x}\right| \phi_{n}^{+}\right\rangle & =-\sin \frac{\varphi_{n}}{2} \sin \frac{\varphi_{n+1}}{2}, \\
\left\langle\phi_{n+1}^{-}\left|\hat{\sigma}_{x}\right| \phi_{n}^{-}\right\rangle & =-\cos \frac{\varphi_{n}}{2} \sin \frac{\varphi_{n+1}}{2},
\end{aligned}
$$

with $\tan \varphi_{n}=2 \lambda \sqrt{n+1} / \Delta$ and $\Delta=\varepsilon-\omega_{0}$. Here we further approximately treat the eigenvalues as $E_{n,+} \approx \omega_{0}(n+1 / 2)+$ $\varepsilon / 2$ and $E_{n,-} \approx \omega_{0}(n+3 / 2)-\varepsilon / 2$. And the corresponding eigenvectors are simplified to $\left|\phi_{n}^{+}\right\rangle \approx|n\rangle \otimes|\uparrow\rangle$ and $\left|\phi_{n}^{-}\right\rangle \approx \mid n+$ $1\rangle \otimes|\downarrow\rangle$. Hence the transition rates upper to the order $(\lambda / \Delta)^{2}$ are given by

$$
\begin{aligned}
& \Gamma_{\mathrm{Q}}^{ \pm}\left(E_{n,+}^{n+1,+}\right) \approx \gamma_{\mathrm{Q}}\left( \pm \omega_{0}\right) n_{\mathrm{Q}}\left( \pm \omega_{0}\right)\left(\frac{\lambda}{\Delta}\right)^{2}(n+1), \\
& \Gamma_{\mathrm{Q}}^{ \pm}\left(E_{n,-}^{n+1,+}\right) \approx \gamma_{\mathrm{Q}}( \pm \varepsilon) n_{\mathrm{Q}}( \pm \varepsilon)\left[1-\left(\frac{\lambda}{\Delta}\right)^{2}(2 n+3)\right] \\
& \Gamma_{\mathrm{Q}}^{ \pm}\left(E_{n,+}^{n+1,-}\right) \approx 0 \\
& \Gamma_{\mathrm{Q}}^{ \pm}\left(E_{n,-}^{n+1,-}\right) \approx \gamma_{\mathrm{Q}}\left( \pm \omega_{0}\right) n_{\mathrm{Q}}\left( \pm \omega_{0}\right)\left(\frac{\lambda}{\Delta}\right)^{2}(n+2)
\end{aligned}
$$

with $E_{n^{\prime}, \sigma^{\prime}}^{n, \sigma}=E_{n, \sigma}-E_{n^{\prime}, \sigma^{\prime}}$. Similarly, the rates related with the R-th bath are approximated by

$$
\begin{aligned}
& \Gamma_{\mathrm{R}}^{ \pm}\left(E_{n,+}^{n+1,+}\right) \approx \gamma_{\mathrm{R}}\left( \pm \omega_{0}\right) n_{\mathrm{R}}\left( \pm \omega_{0}\right)(n+1)\left[1+\left(\frac{\lambda}{\Delta}\right)^{2}\right] \\
& \Gamma_{\mathrm{R}}^{ \pm}\left(E_{n,-}^{n+1,+}\right) \approx \gamma_{\mathrm{R}}( \pm \varepsilon) n_{\mathrm{R}}( \pm \varepsilon)\left(\frac{\lambda}{\Delta}\right)^{2}, \\
& \Gamma_{\mathrm{R}}^{ \pm}\left(E_{n-1,+}^{n,-}\right) \approx 0 \\
& \Gamma_{\mathrm{R}}^{ \pm}\left(E_{n-1,-}^{n,-}\right) \approx \gamma_{\mathrm{R}}\left( \pm \omega_{0}\right) n_{\mathrm{R}}\left( \pm \omega_{0}\right)(n+2)\left[1-\left(\frac{\lambda}{\Delta}\right)^{2}\right]
\end{aligned}
$$


From Eq. (A1) we have the relation at steady state

$$
\begin{aligned}
\sum_{n}[ & \left.\Gamma_{\mathrm{Q}}^{-}\left(E_{n-1,-}^{n,+}\right) P_{n,+}-\Gamma_{\mathrm{Q}}^{+}\left(E_{n-1,-}^{n,+}\right) P_{n-1,-}\right] \\
= & \sum_{n}\left[\Gamma_{\mathrm{R}}^{+}\left(E_{n-1,-}^{n,+}\right) P_{n-1,-}-\Gamma_{\mathrm{R}}^{-}\left(E_{n-1,-}^{n,+}\right) P_{n,+}\right] \\
& +\sum_{n}\left[\Gamma_{\mathrm{Q}}^{+}\left(E_{n-1,+}^{n,+}\right) P_{n-1,+}-\Gamma_{\mathrm{Q}}^{-}\left(E_{n-1,+}^{n,+}\right) P_{n,+}\right] \\
& +\sum_{n}\left[\Gamma_{\mathrm{Q}}^{-}\left(E_{n,+}^{n+1,+}\right) P_{n+1,+}-\Gamma_{\mathrm{Q}}^{+}\left(E_{n,+}^{n+1,+}\right) P_{n,+}\right] .
\end{aligned}
$$

Hence the zeroth-order populations are given by

$$
\begin{aligned}
& P_{n,+}^{(0)} \approx \frac{\left(1-e^{-\beta_{\mathrm{R}} \omega_{0}}\right)}{e^{\beta_{\mathrm{Q}} \varepsilon}+1} e^{-n \beta_{\mathrm{R}} \omega_{0}}, \\
& P_{n,-}^{(0)} \approx \frac{e^{\beta_{\mathrm{Q}} \varepsilon}\left(1-e^{-\beta_{\mathrm{R}} \omega_{0}}\right)}{e^{\beta_{\mathrm{Q}} \varepsilon}+1} e^{-(n+1) \beta_{\mathrm{R}} \omega_{0}},
\end{aligned}
$$

with $\beta_{u}=1 /\left(T_{u}\right)(u=\mathrm{R}, \mathrm{Q})$. Consequently, considering Eqs. (A2) and (A3b) we obtain the leading-order expression of heat current as

$$
J_{\mathrm{Q}}^{x}=\left(\frac{\lambda}{\varepsilon-\omega_{0}}\right)^{2}\left(\omega_{0} I_{x, 1}+\varepsilon I_{x, 2}\right),
$$

where two components are specified as

$$
I_{x, 1}=\gamma_{\mathrm{Q}}\left(\omega_{0}\right)\left\{n_{\mathrm{R}}\left(\omega_{0}\right)\left[1+n_{\mathrm{Q}}\left(\omega_{0}\right)\right]-\left[1+n_{\mathrm{R}}\left(\omega_{0}\right)\right] n_{\mathrm{Q}}\left(\omega_{0}\right)\right\},
$$

$$
I_{x, 2}=\frac{\gamma_{\mathrm{R}}(\varepsilon)}{2 n_{\mathrm{Q}}(\varepsilon)+1}\left\{n_{\mathrm{R}}(\varepsilon)\left[1+n_{\mathrm{Q}}(\varepsilon)\right]-\left[1+n_{\mathrm{R}}(\varepsilon)\right] n_{\mathrm{Q}}(\varepsilon)\right\} \text {. }
$$

\section{3. $\theta=\pi / 2$ case}

At $\theta=\pi / 2$, Based on the eigenbasis $\left|\phi_{n}^{\uparrow(\downarrow)}\right\rangle$ at Eqs. (9a) and (9b) the transition coefficient involved with the Q-th bath at weak qubit-photon coupling is simplified to

$$
\begin{aligned}
\left\langle\phi_{n}^{\sigma}\left|\hat{\sigma}_{x}\right| \phi_{n^{\prime}}^{\bar{\sigma}}\right\rangle \approx & (-1)^{n}\left[\delta_{n, n^{\prime}}+\frac{2 \lambda}{\omega_{0}} \sqrt{n+1} \delta_{n, n^{\prime}-1}\right. \\
& \left.-\frac{2 \lambda}{\omega_{0}} \sqrt{n} \delta_{n, n^{\prime}+1}\right] .
\end{aligned}
$$

Accordingly, the transition rates defined by Eqs. (14a) and (14b) are approximated by

$$
\begin{aligned}
\Gamma_{\mathrm{Q}}^{ \pm}\left(E_{n^{\prime}, \downarrow}^{n, \uparrow}\right) \approx & \delta_{n, n^{\prime}} \kappa_{\mathrm{Q}}^{ \pm}(\varepsilon)+\delta_{n, n^{\prime}-1} n^{\prime}\left(\frac{2 \lambda}{\omega_{0}}\right)^{2} \kappa_{\mathrm{Q}}^{ \pm}\left(\varepsilon-\omega_{0}\right) \\
& +\delta_{n, n^{\prime}+1} n\left(\frac{2 \lambda}{\omega_{0}}\right)^{2} \kappa_{\mathrm{Q}}^{ \pm}\left(\varepsilon+\omega_{0}\right),
\end{aligned}
$$

$$
\Gamma_{\mathrm{Q}}^{ \pm}\left(E_{n^{\prime}, \uparrow}^{n, \downarrow}\right) \approx \delta_{n, n^{\prime}+1} n^{\prime}\left(\frac{2 \lambda}{\omega_{0}}\right)^{2} \kappa_{\mathrm{Q}}^{ \pm}\left(\omega_{0}-\varepsilon\right),
$$

with $\kappa_{\mathrm{Q}}^{+}(\omega)=\gamma_{\mathrm{Q}}(\omega) n_{\mathrm{Q}}(\omega)$ and $\kappa_{\mathrm{Q}}^{-}(\omega)=\gamma_{\mathrm{Q}}(\omega)\left[1+n_{\mathrm{Q}}(\omega)\right]$. Similarly, the transition rates assisted by the R-th thermal bath are given by $\Gamma_{\mathrm{R}}^{+}\left(E_{n-1, \sigma}^{n, \sigma}\right)=\gamma_{\mathrm{R}}\left(\omega_{0}\right) n_{\mathrm{R}}\left(\omega_{0}\right) n$ and $\Gamma_{\mathrm{R}}^{-}\left(E_{n-1, \sigma}^{n, \sigma}\right)=$ $\gamma_{\mathrm{R}}\left(\omega_{0}\right)\left[1+n_{\mathrm{R}}\left(\omega_{0}\right)\right] n$. Hence the zeroth order of populations based on Eq. (A3a) can be directly obtained as

$$
\begin{aligned}
& P_{n, \uparrow}^{(0)} \approx \frac{\left(1-e^{-\beta_{\mathrm{R}} \omega_{0}}\right)}{e^{\beta_{\mathrm{Q}} \varepsilon}+1} e^{-n \beta_{\mathrm{R}} \omega_{0}}, \\
& P_{n, \downarrow}^{(0)} \approx \frac{e^{\beta_{\mathrm{Q}} \varepsilon}\left(1-e^{-\beta_{\mathrm{R}} \omega_{0}}\right)}{e^{\beta_{\mathrm{Q}} \varepsilon}+1} e^{-n \beta_{\mathrm{R}} \omega_{0}} .
\end{aligned}
$$

Moreover, from Eq. (A3b) it is known that

$$
\begin{aligned}
& \left(\frac{2 \lambda}{\omega_{0}}\right)^{2} \sum_{n}\left[\Gamma_{\mathrm{Q}}^{-}\left(E_{n, \downarrow}^{n, \uparrow}\right) P_{n, \uparrow}^{(1)}-\Gamma_{\mathrm{Q}}^{+}\left(E_{n, \downarrow}^{n, \uparrow}\right) P_{n, \downarrow}^{(1)}\right] \\
& \approx \sum_{n}\left[-\Gamma_{\mathrm{Q}}^{-}\left(E_{n-1, \downarrow}^{n, \uparrow}\right) P_{n, \uparrow}^{(0)}+\Gamma_{\mathrm{Q}}^{+}\left(E_{n-1, \downarrow}^{n, \uparrow}\right) P_{n-1, \downarrow}^{(0)}\right. \\
& \quad-\Gamma_{\mathrm{Q}}^{-}\left(E_{n+1, \downarrow}^{n, \uparrow}\right) P_{n, \uparrow}^{(0)}+\Gamma_{\mathrm{Q}}^{+}\left(E_{n+1, \downarrow}^{n, \uparrow}\right) P_{n+1, \downarrow}^{(0)} \\
& \left.\quad-\Gamma_{\mathrm{Q}}^{+}\left(E_{n, \uparrow}^{n+1, \downarrow}\right) P_{n+1, \downarrow}^{(0)}+\Gamma_{\mathrm{Q}}^{-}\left(E_{n, \uparrow}^{n+1, \downarrow}\right) P_{n, \uparrow}^{(0)}\right] .
\end{aligned}
$$

Finally, Then, the current is contributed by three components

$$
J_{\mathrm{Q}}^{z} \approx\left(\frac{2 \lambda}{\omega_{0}}\right)^{2} \omega_{0}\left(I_{z, 1}+I_{z, 2}+I_{z, 3}\right)
$$

where

$$
\begin{aligned}
I_{z, 1}= & \theta\left(\varepsilon+\omega_{0}\right) \gamma_{\mathrm{Q}}\left(\varepsilon+\omega_{0}\right) \frac{1}{2 n_{\mathrm{Q}}(\varepsilon)+1} \\
& \times\left[\left(1+n_{\mathrm{Q}}\left(\varepsilon+\omega_{0}\right)\right) n_{\mathrm{Q}}(\varepsilon) n_{\mathrm{R}}\left(\omega_{0}\right)\right. \\
& \left.-n_{\mathrm{Q}}\left(\varepsilon+\omega_{0}\right)\left(1+n_{\mathrm{Q}}(\varepsilon)\right)\left(1+n_{\mathrm{R}}\left(\omega_{0}\right)\right)\right], \\
I_{z, 2}= & \theta\left(\varepsilon-\omega_{0}\right) \gamma_{\mathrm{Q}}\left(\varepsilon-\omega_{0}\right) \frac{1}{2 n_{\mathrm{Q}}(\varepsilon)+1} \\
& \times\left[n_{\mathrm{Q}}\left(\varepsilon-\omega_{0}\right)\left(1+n_{\mathrm{Q}}(\varepsilon)\right) n_{\mathrm{R}}\left(\omega_{0}\right)\right. \\
& \left.-\left(1+n_{\mathrm{Q}}\left(\varepsilon-\omega_{0}\right)\right) n_{\mathrm{Q}}(\varepsilon)\left(1+n_{\mathrm{R}}\left(\omega_{0}\right)\right)\right], \\
I_{z, 3}= & \theta\left(\omega_{0}-\varepsilon\right) \gamma_{\mathrm{Q}}\left(\omega_{0}-\varepsilon\right) \frac{1}{2 n_{\mathrm{Q}}(\varepsilon)+1} \\
& \times\left[\left(1+n_{\mathrm{Q}}\left(\omega_{0}-\varepsilon\right)\right)\left(1+n_{\mathrm{Q}}(\varepsilon)\right) n_{\mathrm{R}}\left(\omega_{0}\right)\right. \\
& \left.-n_{\mathrm{Q}}\left(\omega_{0}-\varepsilon\right) n_{\mathrm{Q}}(\varepsilon)\left(1+n_{\mathrm{R}}\left(\omega_{0}\right)\right)\right] .
\end{aligned}
$$

with the Heviside function $\theta(x)=1$ for $x \geqslant 0$, and $\theta(x)=0$ for $x<0$.
[1] G. Chen, Nanoscale Energy Transport and Conversion: A Parallel Treatment of Electrons, Molecules, Phonons, and Photons (Oxford University Press, 2005).

[2] H. Xu, D. Mason, L. Y. Jinag, and J. G. E. Harris, Topological energy transfer in an optomechanical system with exceptional points, Nature (London) 537, 80 (2016).
[3] P. Lodahl, S. Mahmoodian, S. Stobbe, A. Rauschenbeute, P. Schneeweiss, J. Volz, H. Pichler, and P. Zoller, Chiral quantum optics, Nature (London) 541, 473 (2017).

[4] A. Ronzani, B. Karimi, J. Senior, Y. C. Chang, J. T. Peltonen, C. D. Chen, and J. P. Pekola, Tunable photonic heat transport in a quantum heat valve, Nat. Phys. 14, 991 (2018). 
[5] D. W. Wang, C. Song, W. Feng, H. Cai, D. Xu, H. Deng, H. K. Li, D. N. Zheng, X. B. Zhu, H. Wang, S. Y. Zhu, and M. O. Scully, Synthesis of antisymmetric spin exchange interaction and chiral spin clusters in superconducting circuits, Nat. Phys. 15, 382 (2019).

[6] K. Micadei, J. P. S. Peterson, A. M. Souza, R. S. Sarthour, Ivan S. Oliveira, G. T. Landi, T. B. Batalhao, R. M. Serra, and E. Lutz, Reversing the direction of heat flow using quantum correlations, Nat. Commun. 10, 2456 (2019).

[7] J. Ren, P. Hanggi, and B. Li, Berry-Phase-Induced Heat Pumping and Its Impact on the Fluctuation Theorem, Phys. Rev. Lett. 104, 170601 (2010).

[8] Z. Wang, L. Q. Wang, J. Z. Wang, C. Wang, and J. Ren, Geometric heat pump: Controlling thermal transport with time-dependent modulations, Front. Phys. 17, 13201 (2022).

[9] Z. Wang, J. Z. Chen, Z. Liu, and J. Ren, Observation of geometric heat pump effect in periodic driven thermal diffusion, arXiv:2110.10001.

[10] G. Kurizki, P. Bertet, Y. Kubo, K. Molmer, D. Petrosyan, P. Rabl, and J. Schmiedmayer, Quantum technologies with hybrid systems, Proc. Natl. Acad. Sci. USA 112, 3866 (2015).

[11] A. Blais, S. M. Girvin, and W. D. Oliver, Quantum information processing and quantum optics with circuit quantum electrodynamics, Nat. Phys. 16, 247 (2020).

[12] A. A. Clerk, K W. Lehnert, P. Bertet, J. R. Petta, and Y. Nakamura, Hybrid quantum systems with circuit quantum electrodynamics, Nat. Phys. 16, 257 (2020).

[13] A. Blais, A. L. Grimsmo, S. M. Girvin, and A. Wallraff, Circuit quantum electrodynamics, Rev. Mod. Phys. 93, 025005 (2021).

[14] I. I. Rabi, On the process of space quantization, Phys. Rev. 49, 324 (1936).

[15] I. I. Rabi, Space quantization in a gyrating magnetic field, Phys. Rev. 51, 652 (1937).

[16] D. Braak, Integrability of the Rabi Model, Phys. Rev. Lett. 107, 100401 (2011).

[17] Q. H. Chen, C. Wang, S. He, T. Liu, and K. L. Wang, Exact solvability of the quantum Rabi model using Bogoliubov operators, Phys. Rev. A 86, 023822 (2012).

[18] P. Forn-Díaz, L. Mamata, E. Rico, J. Kono, and E. Solano, Ultrastrong coupling regimes of light-matter interaction, Rev. Mod. Phys. 91, 025005 (2019).

[19] A. F. Kockum, A. Miranwicz, S. De Liberato, S. Savasta, and F. Nori, Ultrastrong coupling between light and matter, Nat. Rev. Phys. 1, 19 (2019).

[20] A. Le Boité, Theoretical methods for ultrastrong lightmatter interactions, Adv. Quantum Technol. 3, 1900140 (2020).

[21] M. J. Hwang, R. Peubla, and M. B. Pleino, Quantum Phase Transition and Universal Dynamics in the Rabi Model, Phys. Rev. Lett. 115, 180404 (2015).

[22] M. X. Liu, S. Chesi, Z. J. Ying, X. S. Chen, H. G. Luo, and H. Q. Lin, Universal Scaling and Critical Exponents of the Anisotropic Quantum Rabi Model, Phys. Rev. Lett. 119, 220601 (2017).

[23] Y. Y. Zhang, Z. X. Hu, L. B. Fu, H. G. Luo, H. Pu, and X. F. Zhang, Quantum Phases in a Quantum Rabi Triangle, Phys. Rev. Lett. 127, 063602 (2021).

[24] M. L. Cai, Z. D. Liu, W. D. Zhao, Y. K. Wu, Q. X. Mei, Y. Jiang, L. He, X. Zhang, Z. C. Zhou, and L. M. Duan, Observation of a quantum phase transition in the quantum
Rabi model with a single trapped ion, Nat. Commun. 12, 1126 (2021).

[25] L. Garziano, R. Stassi, V. Macrí, A. F. Kockum, S. Savasta, and F. Nori, Multiphoton quantum Rabi oscillations in ultrastrong cavity QED, Phys. Rev. A 92, 063830 (2015).

[26] L. Garziano, V. Macrí, R. Stassi, O. Di Stefano, F. Nori, and S. Savasta, One Photon can Simultaneously Excite Two or More Atoms, Phys. Rev. Lett. 117, 043601 (2016).

[27] X. Wang, A. Miranowicz, H. R. Li, and F. Nori, Observing pure effects of counter-rotating terms without ultrastrong coupling: A single photon can simultaneously excite two qubits, Phys. Rev. A 96, 063820 (2017).

[28] V. Macrí, F. Minganti, A. F. Kockum, A. Ridolfo, S. Savasta, F. Nori, Revealing higher-order light and matter energy exchanges using quantum trajectories in ultrastrong coupling, arXiv:2107.08759.

[29] J. H. Wang, Z. Q. Wu, and J. Z. He, Quantum Otto engine of a two-level atom with single-mode fields, Phys. Rev. E 85, 041148 (2012).

[30] F. Altintas, A. Ü. C Hardal, and Ö. E. Müstecaplioglu, Rabi model as a quantum coherent heat engine: From quantum biology to superconducting circuits, Phys. Rev. A 91, 023816 (2015)

[31] S. Seah, S. Nimmrichter, and V. Scarani, Refrigeration beyond weak internal coupling, Phys. Rev. E 98, 012131 (2018).

[32] J. Senior, A. Gubaydullin, B. Karimi, J. T. Peltonen, J. Ankerhold, and J. P. Pekola, Heat rectification via a superconducting artificial atom, Comm. Phys. 3, 40 (2020).

[33] M. Majland, K. S. Christensen, and N. T. Zinner, Quantum thermal transistor in superconducting circuits, Phys. Rev. B 101, 184510 (2020).

[34] J. P. Pekola and B. Karimi, Colloquium: Quantum heat transport in condensed matter systems, Rev. Mod. Phys. 93, 041001 (2021).

[35] Y. Lu, N. Lambert, A. F. Kockum, K. Funo, A. Bengtsson, S. Gasparinetti, F. Nori, and P. Delsing, Nonequilibrium heat transport and work with a single artificial atom coupled to a waveguide: emission without external driving, arXiv:2107.12700.

[36] A. Gubaydullin, G. Thomas, D. S. Golubev, D. Lvov, J. T. Peltonen, and J. P. Pekola, Photonic heat transport in three terminal superconducting circuit, arXiv:2112.09224.

[37] J. Iles-Smith, N. Lambert, and A. Nazir, Environmental dynamics, correlations, and the emergence of noncanonical equilibrium states in open quantum systems, Phys. Rev. A 90, 032114 (2014).

[38] J. Iles-Smith, A. G. Dijkstra, N. Lambert, and A. Nazir, Energy transfer in structured and unstructured environments: Master equations beyond the Born-Markov approximations, J. Chem. Phys. 144, 044110 (2016).

[39] H. Maguire, J. Iles-Smith, and A. Nazir, Environmental nonadditivity and Franck-Condon Physics in Nonequilibrium Quantum Systems, Phys. Rev. Lett. 123, 093601 (2019).

[40] T. Yamamoto and T. Kato, Heat transport through a two-level system embedded between two harmonic resonators, J. Phys.: Condens. Matter 33, 395303 (2021).

[41] C. Wang, L. Q. Wang, and J. Ren, Managing quantum heat transfer in a nonequilibrium qubit-phonon hybrid system with coherent phonon states, Chin. Phys. Lett. 38, 010501 (2021). 
[42] C. Wang, L. Q. Wang, and J. Ren, Impact of counter-rotatingwave term on quantum heat transfer and phonon statistics in nonequilibrium qubit-phonon hybrid system, Chin. Phys. B 30, 030506 (2021).

[43] C. Wang, H. Chen, and J. Q. Liao, Nonequilibrium thermal transport and photon squeezing in a quadratic qubit-resonator system, Phys. Rev. A 104, 033701 (2021).

[44] A. Imamoglu, H. Schmidt, G. Woods, and M. Deutsch, Strongly Interacting Photons in a Nonlinear Cavity, Phys. Rev. Lett. 79, 1467 (1997).

[45] K. M. Birnbaum, A. Boca, R. Miller, A. D. Boozer, T. E. Northup, and H. J. Kimble, Photon blockade in an optical cavity with one trapped atom, Nature (London) 436, 87 (2005).

[46] H. J. Carmichael, Statistical Methods in Quantum Optics 1, Master Equations and Fokker Planck Equations (Springer, 1999).

[47] R. J. Glauber, The quantum theory of optical coherence, Phys. Rev. 130, 2529 (1963).

[48] J. H. Eberly and N. E. Rehler, Superradiant intensity fluctuations, Phys. Rev. A 2, 1607 (1970).

[49] R. Bonifacio, P. Schwendimann, and Fritz Haake, Quantum statistical theory of superradiance. I, Phys. Rev. A 4, 302 (1971).

[50] R. Bonifacio, P. Schwendimann, and Fritz Haake, Quantum statistical theory of superradiance. II, Phys. Rev. A 4, 854 (1971).

[51] D. Meiser and M. J. Holland, Intensity fluctuations in steadystate superradiance, Phys. Rev. A 81, 063827 (2010).

[52] A. Aufféves, D. Gerace, S. Portolan, A. Drezet, and M. F. Santos, Few emitters in a cavity: from cooperative emission to individualization, New J. Phys. 13, 093020 (2011).

[53] E. Mascarenhas, D. Gerace, M. F. Santos, and A. Aufféves, Cooperativity of a few quantum emitters in a single-mode cavity, Phys. Rev. A 88, 063825 (2013).

[54] D. Roy, C. M. Wilson, and O. Firstenberg, Colloquium: Strongly interacting photons in one-dimensional continuum, Rev. Mod. Phys. 89, 021001 (2017).

[55] T. Fink, A. Schade, S. Höfling, C. Schneider, and A. Imamoglu, Signatures of a dissipative phase transition in photon correlation measurements, Nat. Phys. 14, 365 (2018).

[56] P. Rabl, Photon Blockade Effect in Optomechanical Systems, Phys. Rev. Lett. 107, 063601 (2011).

[57] A. Ridolfo, M. Leib, S. Savasta, and M. J. Hartmann, Photon Blockade in the Ultrastrong Coupling Regime, Phys. Rev. Lett. 109, 193602 (2012).

[58] A. Ridolfo, S. Savasta, and M. J. Hartmann, Nonclassical Radiation from Thermal Cavities in the Ultrastrong Coupling Regime, Phys. Rev. Lett. 110, 163601 (2013).

[59] R. Stassi, A. Ridolfo, O. Di Stefano, M. J. Hartmann, and S. Savasta, Spontaneous Conversion from Virtual to Real Photons in the Ultrastrong-Coupling Regime, Phys. Rev. Lett. 110, 243601 (2013).

[60] L. Garziano, A. Ridolfo, S. De Liberato, and S. Savasta, Cavity QED in the ultrastrong coupling regime: Photon bunching from the emission of individual dressed qubits, ACS. Photonics 4, 2345 (2017).

[61] Q. Schaeverbeke, R. Avriller, T. Frederiksen, and F. Pistolesi, Single-Photon Emission Mediated by Single-Electron Tunneling in Plasmonic Nanojunctions, Phys. Rev. Lett. 123, 246601 (2019).
[62] Q. Bin, X. Y. Lü, F. P. Laussy, F. Nori, and Y. Wu, N-Phonon Bundle Emission via the Stokes Process, Phys. Rev. Lett. 124, 053601 (2020).

[63] Q. Bin, Y. Wu, and X. Y. Lü, Parity-Symmetry-Protected Multiphoton Bundle Emission, Phys. Rev. Lett. 127, 073602 (2021).

[64] T. Niemczyk, F. Deppe, H. Huebl, E. P. Menzel, F. Hocke, M. J. Schwarz, J. J. Garcia-Ripoll, D. Zueco, T. Hümmer, E. Solano, A. Marx, and R. Gross, Circuit quantum electrodynamics in the ultrastrong-coupling regime, Nat. Phys. 6, 772 (2010).

[65] F. Yoshihara, T. Fuse, S. Ashhab, K. Kakuyanagi, S. Saito, and K. Semba, Superconducting qubit"Coscillator circuit beyond the ultrastrong-coupling regime, Nat. Phys. 13, 44 (2017).

[66] Y. J. Zhao, Y. L. Liu, Y. X. Liu, and F. Nori, Generating nonclassical photon states via longitudinal couplings between superconducting qubits and microwave fields, Phys. Rev. A 91, 053820 (2015).

[67] X. Wang, A. Miranowicz, H. R. Li, and F. Nori, Multipleoutput microwave single-photon source using superconducting circuits with longitudinal and transverse couplings, Phys. Rev. A 94, 053858 (2016).

[68] M. T. Naseem and Ö. E. Müstecaplioğlu, Antibunching via cooling by heating, Phys. Rev. A 105, 012201 (2022).

[69] P. M. Billangeon, J. S. Tsai, and Y. Nakamura, CircuitQED-based scalable architectures for quantum information processing with superconducting qubits, Phys. Rev. B 91, 094517 (2015).

[70] S. Richer and D. DiVincenzo, Circuit design implementing longitudinal coupling: A scalable scheme for superconducting qubits, Phys. Rev. B 93, 134501 (2016).

[71] N. Didier, J. Bourassa, and A Blais, Fast Quantum Nondemolition Readout by Parametric Modulation of Longitudinal Qubit-Oscillator Interaction, Phys. Rev. Lett. 115, 203601 (2015).

[72] A. L. Grimsmo and T. B. Smith, Majorana qubit readout using longitudinal qubit-resonator interaction, Phys. Rev. B 99, 235420 (2019).

[73] N. Lambert, M. Cirio, M. Delbecq, G. Allison, M. Marx, S. Tarucha, and F. Nori, Amplified and tunable transverse and longitudinal spin-photon coupling in hybrid circuit-QED, Phys. Rev. B 97, 125429 (2018).

[74] M. Cattaneo and G. S. Paraoanu, Engineering dissipation with resistive elements in circuit quantum electrodynamics, Adv. Quantum Technol. 4, 2100054 (2021).

[75] A. A. Clerk, M. H. Devoret, S. M. Girvin, F. Marquardt, and R. J. Schoelkopf, Introduction to quantum noise, measurement, and amplification, Rev. Mod. Phys. 82, 1155 (2010).

[76] J. Hausinger and M. Grifoni, Dissipative dynamics of a biased qubit coupled to a harmonic oscillator: analytical results beyond the rotating wave approximation, New J. Phys. 10, 115015 (2008).

[77] L. Magazzù and M. Grifoni, Transmission spectra of an ultrastrongly coupled qubit-dissipative resonator system, J. Stat. Mech. (2019) 104002.

[78] L. Magazzù, P. Forn-Díaz, and M. Grifoni, Transmission spectra of the driven, dissipative Rabi model in the ultrastrongcoupling regime, Phys. Rev. A 104, 053711 (2021).

[79] J. Larson, Integrability vs quantum thermalization, J. Phys. B: At. Mol. Opt. Phys. 46, 224016 (2013). 
[80] Y. Y. Zhang, Q. H. Chen, and Y. Zhao, Generalized rotatingwave approximation to biased qubit-oscillator systems, Phys. Rev. A 87, 033827 (2013).

[81] Q. T. Xie, H. H. Zhong, M. T. Batchelor, and C. H. Lee, The quantum Rabi model: solution and dynamics, J. Phys. A: Math. Theor. 50, 113001 (2017).

[82] F. Beaudoin, J. M. Gambetta, and A. Blais, Dissipation and ultrastrong coupling in circuit QED, Phys. Rev. A 84, 043832 (2011).

[83] A. Settineri, V. Macrí, A. Ridolfo, O. Di Stefano, A. F. Kockum, F. Nori, and S. Savasta, Dissipation and thermal noise in hybrid quantum systems in the ultrastrong-coupling regime, Phys. Rev. A 98, 053834 (2018).

[84] B. Li, L. Wang, and G. Casati, Negative differential thermal resistance and thermal transistor, Appl. Phys. Lett. 88, 143501 (2006).

[85] N. B. Li, J. Ren, L. Wang, G. Zhang, P. Hänggi, and B. Li, Colloquium: Phononics: Manipulating heat flow with electronic analogs and beyond, Rev. Mod. Phys. 84, 1045 (2012).

[86] D. H. He, S. Buyukdagli, and B. Hu, Origin of negative differential thermal resistance in a chain of two weakly coupled nonlinear lattices, Phys. Rev. B 80, 104302 (2009).

[87] D. H. He, B. Q. Ai, H. K. Chan, and B. Hu, Heat conduction in the nonlinear response regime: Scaling, boundary jumps, and negative differential thermal resistance, Phys. Rev. E 81, 041131 (2010).

[88] H. K. Chan, D. H. He, and B. Hu, Scaling analysis of negative differential thermal resistance, Phys. Rev. E 89, 052126 (2014).

[89] Q. H. Chen, Y. Y. Zhang, T. Liu, and K. L. Wang, Numerically exact solution to the finite-size Dicke model, Phys. Rev. A 78, 051801(R) (2008).

[90] J. Ren, J. X. Zhu, J. E. Gubernatis, C. Wang, and B. Li, Thermoelectric transport with electron-phonon coupling and electron-electron interaction in molecular junctions, Phys. Rev. B 85, 155443 (2012).

[91] T. Ruokola and T. Ojanen, Thermal conductance in a spinboson model: Cotunneling and low-temperature properties, Phys. Rev. B 83, 045417 (2011).
[92] C. Wang, J. Ren, and J. Cao, Nonequilibrium energy transfer at nanoscale: A unified theory from weak to strong coupling, Sci. Rep. 5, 11787 (2015).

[93] D. Z. Xu and J. Cao, Non-canonical distribution and nonequilibrium transport beyond weak system-bath coupling regime: A polaron transformation approach, Front. Phys. 11, 110308 (2016).

[94] C. Wang, J. Ren, and J. Cao, Unifying quantum heat transfer in a nonequilibrium spin-boson model with full counting statistics, Phys. Rev. A 95, 023610 (2017).

[95] T. Q. Chen, V. Balachandran, C. Guo, and D. Poletti, Steadystate quantum transport through an anharmonic oscillator strongly coupled to two heat reservoirs, Phys. Rev. E 102, 012155 (2020).

[96] C. R. Du, C. Y. Hsieh, J. J. Liu, J. L. Wu, and J. Cao, Unusual transport properties with noncommutative system-bath coupling operators, J. Phys. Chem. Lett. 11, 4080 (2020).

[97] J. Ren and J. X. Zhu, Heat diode effect and negative differential thermal conductance across nanoscale metal-dielectric interfaces, Phys. Rev. B 87, 241412(R) (2013).

[98] J. Ren, Predicted rectification and negative differential spin Seebeck effect at magnetic interfaces, Phys. Rev. B 88, 220406(R) (2013).

[99] J. H. Jiang, M. Kulkarni, D. Segal, and Y. Imry, Phonon thermoelectric transistors and rectifiers, Phys. Rev. B 92, 045309 (2015).

[100] J. H. Jiang and Y. Imry, Enhancing Thermoelectric Performance Using Nonlinear Transport Effects, Phys. Rev. Applied 7, 064001 (2017).

[101] J. C. Lu, R. Q. Wang, J. Ren, M. Kulkarni, and J. H. Jiang, Quantum-dot circuit-QED thermoelectric diodes and transistors, Phys. Rev. B 99, 035129 (2019).

[102] J. C. Lu, R. Q. Wang, C. Wang, and J. H. Jiang, Brownian thermal transistors and refrigerators in mesoscopic systems, Phys. Rev. B 102, 125405 (2020).

[103] J. C. Lu, J. H. Jiang, and Y. Imry, Unconventional fourterminal thermoelectric transport due to inelastic transport: Cooling by transverse heat current, transverse thermoelectric effect, and Maxwell demon, Phys. Rev. B 103, 085429 (2021). 